\title{
Estimation and Direct Equalization of Doubly Selective Channels
}

\author{
Imad Barhumi, ${ }^{1}$ Geert Leus, ${ }^{2}$ and Marc Moonen ${ }^{3}$ \\ ${ }^{1}$ Electrical Engineering Department, United Arab Emirates University, Al-Ain 17555, United Arab Emirates \\ ${ }^{2}$ Faculty of Electrical Engineering, Mathematics, and Computer Science, Delft University of Technology, \\ Mekelweg 4, 2628CD Delft, The Netherlands \\ ${ }^{3}$ ESAT/SCD-SISTA, Katholieke Universiteit Leuven, Kasteelpark Arenberg 10, 3001 Leuven, Belgium
}

Received 15 June 2005; Revised 9 June 2006; Accepted 13 August 2006

\begin{abstract}
We propose channel estimation and direct equalization techniques for transmission over doubly selective channels. The doubly selective channel is approximated using the basis expansion model (BEM). Linear and decision feedback equalizers implemented by time-varying finite impulse response (FIR) filters may then be used to equalize the doubly selective channel, where the time-varying FIR filters are designed according to the BEM. In this sense, the equalizer BEM coefficients are obtained either based on channel estimation or directly. The proposed channel estimation and direct equalization techniques range from pilot-symbol-assistedmodulation- (PSAM-) based techniques to blind and semiblind techniques. In PSAM techniques, pilot symbols are utilized to estimate the channel or directly obtain the equalizer coefficients. The training overhead can be completely eliminated by using blind techniques or reduced by combining training-based techniques with blind techniques resulting in semiblind techniques. Numerical results are conducted to verify the different proposed channel estimation and direct equalization techniques.
\end{abstract}

Copyright (c) 2006 Imad Barhumi et al. This is an open access article distributed under the Creative Commons Attribution License, which permits unrestricted use, distribution, and reproduction in any medium, provided the original work is properly cited.

\section{INTRODUCTION}

Over the last decade, the mobile wireless telecommunication industry has undergone tremendous changes and experienced rapid growth. The reason behind this growth is the increasing demand for bandwidth hungry multimedia applications. This demand for even higher data rates at the user's terminal is expected to continue for the coming years as more and more applications are emerging. Therefore, current cellular systems have been designed to provide date rates that range from a few megabits per second for stationary or low mobility users to a few hundred kilobits per second for high mobility users. In addition to the frequency-selectivity characteristics caused by multipath propagation, the channel often exhibits time-variant characteristics caused by the user's mobility. This results in the so-called doubly selective (timeand frequency-selective) channels.

In $[1,2]$, linear and decision feedback equalizers have been developed for single carrier transmission over doubly selective channels. There, the time-varying channel was approximated using the basis expansion model (BEM). The BEM coefficients are then used to design the equalizer (linear or decision feedback). So far, it was assumed that the BEM coefficients are perfectly known at the receiver, and that they were obtained by a least-squares (LS) fitting to the noiseless underlying communication channel (modeled using Jakes' model). In other words, perfect channel state information (CSI) was assumed to be known at the receiver side. This is, however, far from being realistic, since a more realistic approach is to estimate the channel or directly obtain the equalizer coefficients. This can be achieved by using training symbols, or blindly or semiblindly by combining training with blind techniques. In this paper we will focus on pilot-symbol-assisted-modulation- (PSAM-) based, blind, and semiblind techniques for channel estimation and direct equalization of rapidly time-varying channels.

PSAM techniques rely on time multiplexing data symbols and known pilot symbols at known positions, which the receiver utilizes to either estimate the channel or obtain the equalizer coefficients directly. In this context, we first derive the optimal minimum mean-squared error (MMSE) interpolation filter. Then we derive the conventional BEM channel estimation technique based on LS fitting. While the MMSE interpolation filter requires the channel statistics, the latter does not require a priori knowledge of the channel statistics. It was shown in $[3,4]$ that the modeling error between the true channel and the BEM channel model is quite large for the case when the BEM period equals the time window. 
This case corresponds to a critical sampling of the Doppler spectrum. Reducing this modeling error can be achieved by setting the BEM period equal to a multiple of the time window [5]. In other words, we can reduce the modeling error by oversampling the Doppler spectrum. In [6] the authors treated the first case ignoring the modeling error. However, when BEM oversampling is used, LS fitting of the BEM channel based on pilot symbols only is sensitive to noise. Here, we show that robust-PSAM-based channel estimation can be obtained by combining the optimal-MMSE-interpolationbased channel estimation with the LS fitting of the BEM. Although this can be applied to the critically sampled case as well as to the oversampled case with oversampling factor greater than one, little gain is obtained for the critically sampled case. In addition, we show that the channel estimation step can be skipped and obtain the equalizer coefficients directly based on the pilot symbols. This is referred to as PSAM-based direct equalization.

The training overhead imposed on the system can be completely eliminated by using blind techniques for channel estimation and direct equalization. Due to the poor performance of blind techniques and their high implementation complexity, better performance and reduced complexity semiblind techniques can be obtained. Semiblind techniques are obtained by combining blind techniques with training.

For our blind techniques we focus on deterministic approaches. For time-invariant (TI) channels, a least-squaresbased deterministic channel estimation method is discussed in [7], and deterministic mutually referenced equalization is proposed in $[8,9]$. Subspace-based methods have also been proposed for channel identification/equalization for TI channels [10-15]. For doubly selective channels, deterministic blind identification/equalization techniques are proposed in $[16,17]$, where for a zero-forcing (ZF) FIR solution to exist, the number of subchannels (receive antennas) is required to be greater than the number of basis functions used for BEM channel modeling. In $[18,19]$ blind techniques based on linear prediction are proposed for doubly selective channels, where second-order statistics of the data are used. However, these techniques also require the number of receive antennas to be greater than the number of basis functions of the BEM channel. However, we propose an approach for which the ZF solution already exists when only two subchannels (receive antennas) are used.

This paper is organized as follows. In Section 2, the system model is introduced. PSAM techniques are introduced in Section 3. In Section 4, blind and semiblind techniques are investigated. Simulation results are given in Section 5. Finally our conclusions are drawn in Section 6.

\section{Notations}

We use upper (lower) bold face letters to denote matrices (column vectors). Superscripts $*, T, H$, and $\dagger$ represent conjugate, transpose, Hermitian, and pseudo-inverse, respectively. Continuous-time variables (discrete-time) are denoted as $x(\cdot)(x[\cdot]) . \mathcal{E}\{\cdot\}$ denotes expectation. We denote the $N \times N$ identity matrix as $\mathbf{I}_{N}$, the $M \times N$ all-zero matrix as
$\mathbf{0}_{M \times N}$, and the $M \times N$ all-one matrix as $\mathbf{1}_{M \times N}$. Finally, $\operatorname{diag}\{\mathbf{x}\}$ denotes the diagonal matrix with vector $\mathbf{x}$ on its diagonal.

\section{SYSTEM MODEL}

We assume a single-input multiple-output (SIMO) system with $N_{r}$ receive antennas. Focusing on a baseband-equivalent description, the transmitted signal consists of discrete symbols that are pulse shaped with the transmit filter $g_{\operatorname{tr}}(t)$ and transmitted at a rate of $1 / T$ symbols per second (the symbol rate). Hence, the baseband transmitted signal can be written as

$$
x(t)=\sum_{k=-\infty}^{\infty} x[k] g_{\operatorname{tr}}(t-k T)
$$

where $x[k]$ is the $k$ th transmitted QAM symbol. The received signal, on the other hand, is filtered with the receive filter $g_{\text {rec }}(t)$. Assuming the channel time-variation is negligible over the time span of the receive filter, the input-output relationship can be written as

$$
\begin{aligned}
& y^{(r)}(t) \\
& \quad=\sum_{k=-\infty}^{\infty} x[k] \iint_{-\infty}^{\infty} g^{(r)}(t ; \tau) g_{\text {tr }}(t-k T-\tau-s) g_{\text {rec }}(s) \mathrm{d} s \mathrm{~d} \tau \\
& \quad+v^{(r)}(t),
\end{aligned}
$$

where $g^{(r)}(t ; \tau)$ is the doubly selective channel characterizing the link between the transmitter and the $r$ th receive antenna, and $v^{(r)}(t)$ is the baseband equivalent additive noise at the $r$ th receive antenna. The received signal is then sampled at the symbol rate $1 / T .{ }^{1}$ Defining $y^{(r)}[n]=y^{(r)}(n T)$, the discretetime input-output relationship can be written as

$$
\begin{aligned}
y^{(r)}[n]= & \sum_{k=-\infty}^{\infty} x[k] \iint_{-\infty}^{\infty} g^{(r)}(n T ; \tau) \\
& \quad \times g_{\operatorname{tr}}((n-k) T-\tau-s) g_{\text {rec }}(s) \mathrm{d} s \mathrm{~d} \tau \\
& +v^{(r)}(n T) \\
= & \sum_{k=-\infty}^{\infty} x[k] g^{(r)}[n ; n-k]+v^{(r)}[n]
\end{aligned}
$$

where $g^{(r)}[n ; n-k]$ is the discrete-time impulse response of the doubly selective channel characterizing the link between the transmitter and the $r$ th receive antenna, and $v^{(r)}[n]$ is the discrete-time additive noise at the $r$ th receive antenna.

\footnotetext{
1 Temporal oversampling is also possible here to obtain a SIMO system. In this paper we consider the use of multiple receive antennas. Assuming temporal oversampling, to some degree, is equivalent to using multiple receive antennas, where the number of receive antennas is equal to the temporal oversampling factor.
} 
For causal doubly selective channels of order $L$, the inputoutput relationship (3) can be written as

$$
y^{(r)}[n]=\sum_{l=0}^{L} g^{(r)}[n ; l] x[n-l]+v^{(r)}[n] .
$$

\section{Basis expansion channel model}

The mobile wireless channel can be characterized as a timevarying multipath fading channel, where each resolvable path consists of a superposition of a large number of independent scatterers (rays) that arrive at the receiver almost simultaneously. This is referred to as Jakes' channel model [20]. In this model the variation of each tap can be simulated as

$$
g^{(r)}[n ; l]=\sum_{\mu=0}^{Q_{J}-1} G_{l, \mu}^{(r)} e^{j 2 \pi f_{\max } T \cos \phi_{l, \mu}^{(r)} n}
$$

where $Q_{J}$ is the number of scattering rays, $G_{l, \mu}^{(r)}$ is the complex gain, $\phi_{l, \mu}^{(r)}$ is the angle of arrival of the $\mu$ th ray of the $l$ th tap, respectively, and $f_{\max }$ is the maximum Doppler spread. $G_{l, \mu}^{(r)}$ are independent identically distributed (i.i.d) complex Gaussian random variables with zero mean and variance $\sigma_{l}^{2} /\left(2 Q_{J}\right)$ per dimension, where $\sigma_{l}^{2}$ is the $l$ th tap power, and $\phi_{l, \mu}^{(r)}$ are i.i.d. random variables uniformly distributed over $[0,2 \pi]$. Note that the model in (5) implies the wide sense stationarity (WSS), where the channel correlation function is invariant over time.

The channel model in (5) has a rather complex structure due to the large (possibly infinite) number of parameters to be identified, which complicates, if not prevents, the development of low complexity equalizers. This motivates the use of alternative models that have fewer parameters. This is the motivation behind the basis expansion model (BEM) $[16,21-23]$. In this BEM, the time-varying channel $g^{(r)}[n ; l]$ over a window of $N$ samples is expressed as a superposition of complex exponential basis functions with frequencies on a discrete Fourier transform (DFT) grid. In other words, the time-varying channel $g^{(r)}[n, l]$ is approximated for $n \in\{0, \ldots, N-1\}$ by a BEM as

$$
h^{(r)}[n ; l]=\sum_{q=-Q / 2}^{Q / 2} h_{q, l}^{(r)} e^{j 2 \pi q n / K}
$$

where $(Q+1)$ is the number of basis functions, and $K$ is the BEM period. $Q$ and $K$ should be chosen such that $\mathrm{Q} /(2 K T)$ is larger than the maximum Doppler frequency, that is, $Q /(2 K T) \geq f_{\max }$. Finally, $h_{q, l}^{(r)}$ is the coefficient of the $q$ th basis of the $l$ th tap of the time-varying channel characterizing the link between the transmitter and the $r$ th receive antenna, which is kept invariant over a period of NT, but may

\begin{tabular}{|c|c|c|c|c|c|}
\hline \multicolumn{2}{|c|}{ Training } & \multirow[t]{2}{*}{ Data } & \multicolumn{2}{|c|}{ Training } & Data \\
\hline $0_{1 \times L}$ & $0_{1 \times L}$ & & $0_{1 \times L}$ & $0_{1 \times L}$ & \\
\hline
\end{tabular}
change from block to block. The BEM coefficients $h_{q, l}^{(r)}$ may be approximated as complex Gaussian random variables.
FIGURE 1: Optimal training for doubly selective channels.

\section{PSAM TECHNIQUES}

\subsection{PSAM channel estimation}

For the sake of simplicity we assume the number of receive antennas $N_{r}=1$, that is, we assume a SISO system. This is a valid assumption because we can decouple the SIMO channel estimation problem into $N_{r}$ parallel SISO channel estimation problems. Using the time-domain training procedure proposed in $[6,24]$, the doubly selective channel of order $L$ can be viewed as $L$ flat fading channels on the part of the received sequence that corresponds to training. The data/training multiplexing is shown in Figure 1, where the training part consists of a training symbol surrounded by $L$ zeros on each side. Assuming we use $P$ such training clusters where the pilot symbols are located at positions $n_{0}, \ldots, n_{P-1}$, the input-output relation on the pilot positions can be written as

$$
y\left[n_{p, l}\right]=g\left[n_{p, l} ; l\right] x\left[n_{p}\right]+v\left[n_{p, l}\right],
$$

where $n_{p, l}=n_{p}+l$ for $l=0, \ldots, L$.

Define $\quad \mathbf{y}_{t, l}=\left[y\left[n_{0, l}\right], \ldots, y\left[n_{P-1, l}\right]\right]^{T}, \quad \mathbf{X}_{t}=$ $\operatorname{diag}\left\{\left[x\left[n_{0}\right], \ldots, x\left[n_{P-1}\right]\right]^{T}\right\}, \mathbf{g}_{t, l}=\left[g\left[n_{0} ; l\right], \ldots, g\left[n_{P-1} ; l\right]\right]^{T}$, and $\mathbf{v}_{t, l}=\left[v\left[n_{0, l}\right], \ldots, v\left[n_{P-1, l}\right]\right]^{T}$, the input-output relation in (7) can now be written in vector form as

$$
\mathbf{y}_{t, l}=\mathbf{X}_{t} \mathbf{g}_{t, l}+\mathbf{v}_{t, l}
$$

In this section, we first derive the optimal minimum mean-squared error (MMSE) PSAM-based channel estimation, which leads to the development of the optimal interpolation filter. However, since the BEM coefficients of the time-varying channel are needed to design the equalizers (linear and decision feedback), the PSAM-based estimation of the BEM coefficients is also discussed and combined with PSAM-based MMSE channel estimation to enhance the LS fitting of the true channel and the estimated one.

\subsubsection{MMSE channel estimation}

From (8), an estimate of the $l$ th tap of the time-varying channel $\hat{\mathbf{g}}_{l}=[\hat{g}[0 ; l], \ldots, \hat{g}[N-1 ; l]]^{T}$ is obtained by applying a $P \times N$ interpolation matrix $\mathbf{W}_{l}$ as

$$
\hat{\mathbf{g}}_{l}=\mathbf{W}_{l}^{H} \mathbf{y}_{t, l}
$$

Define the mean-squared error cost function $g$ as

$$
\mathcal{g}\left(\mathbf{W}_{l}\right)=\mathscr{E}\left\|\mathbf{g}_{l}-\mathbf{W}_{l}^{H} \mathbf{y}_{t, l}\right\|^{2}
$$

where $\mathbf{g}_{l}=[g[0 ; l], \ldots, g[N-1 ; l]]^{T}$ is the channel state information at the $l$ th tap. 
The MMSE interpolation matrix $\mathbf{W}_{l}$ is then obtained by solving

$$
\min _{\mathbf{W}_{l}} \mathfrak{g} .
$$

Minimizing this cost function, we obtain [25]

$$
\mathbf{W}_{\mathrm{MMSE}, l}=\left(\mathbf{X}_{t} \mathbf{R}_{p, l} \mathbf{X}_{t}^{*}+\mathbf{R}_{\widetilde{v}}\right)^{-1} \mathbf{X}_{t} \mathbf{R}_{g, l}
$$

where $\mathbf{R}_{p, l}$ is the $l$ th tap channel correlation matrix on the pilots given by

$$
\begin{aligned}
& \mathbf{R}_{p, l} \\
& =\left[\begin{array}{cccc}
r_{g, l}[0] & r_{g, l}\left[n_{0}-n_{1}\right] & \cdots & r_{g, l}\left[n_{0}-n_{P-1}\right] \\
r_{g, l}\left[n_{1}-n_{0}\right] & r_{g, l}[0] & \cdots & r_{g, l}\left[n_{1}-n_{P-1}\right] \\
\vdots & & \ddots & \vdots \\
r_{g, l}\left[n_{P-1}-n_{0}\right] & r_{g, l}\left[n_{P-1}-n_{1}\right] & \cdots & r_{g, l}[0]
\end{array}\right],
\end{aligned}
$$

and $\mathbf{R}_{g, l}$ is given by

$$
\mathbf{R}_{g, l}=\left[\begin{array}{cccc}
r_{g, l}\left[n_{0}\right] & r_{g, l}\left[n_{0}-1\right] & \cdots & r_{g, l}\left[n_{0}-N+1\right] \\
r_{g, l}\left[n_{1}\right] & r_{g, l}\left[n_{1}-1\right] & \cdots & r_{g, l}\left[n_{1}-N+1\right] \\
\vdots & & \ddots & \vdots \\
r_{g, l}\left[n_{P-1}\right] & r_{g, l}\left[n_{P-1}-1\right] & \cdots & r_{g, l}\left[n_{P-1}-N+1\right]
\end{array}\right],
$$

with $r_{g, l}[k]=\mathscr{E}\left\{g[n ; l] g^{*}[n-k ; l]\right\} . \mathbf{R}_{\tilde{v}}$ is the covariance matrix of the channel estimation error at the pilot positions. Both $\mathbf{R}_{p, l}$ and $\mathbf{R}_{g, l}$ are assumed to be known (assuming Jakes' model, then it only requires the knowledge of the system maximum Doppler shift $f_{\max }$ and the power delay profile). Assuming i.i.d input symbols $x[n]$, the training is of Kronecker delta form (i.e., $x\left[n_{p}\right]=1 \forall p=0, \ldots, P-1$ ), and white noise with normalized power $\beta$, then $\mathbf{R}_{\widetilde{v}}=\beta \mathbf{I}_{P}$. The MMSE interpolation matrix on the $l$ th tap $\mathbf{W}_{\mathrm{MMSE}, l}$ can now be written as

$$
\mathbf{W}_{\mathrm{MMSE}, l}=\left(\mathbf{R}_{p, l}+\beta \mathbf{I}_{P}\right)^{-1} \mathbf{R}_{g, l} .
$$

Note that for channels with uniform power delay profile, the matrices $\mathbf{R}_{P, l}, \mathbf{R}_{g, l}$, and $\mathbf{W}_{\mathrm{MMSE}, l}$ are identical and independent of $l$, which means that they need to be computed once.

\subsubsection{BEM channel estimation}

For time-varying FIR equalization, where the time-varying FIR equalizers are designed according to the BEM, the BEM coefficients of the time-varying channel are then required to design these equalizers. To this end, we define $\mathbf{h}_{l}=$ $\left[h_{-Q / 2, l}, \ldots, h_{Q / 2, l}\right]^{T}$ as the vector containing the BEM coefficients of the lth tap of the time-varying channel. In the ideal case, where the time-varying channel $\mathbf{g}_{l}$ is perfectly known at the receiver, a LS fit of the BEM to the time-varying channel model can be obtained by solving

$$
\min _{\mathbf{h}_{l}}\left\|\mathbf{g}_{l}-\mathcal{L} \mathbf{h}_{l}\right\|^{2}
$$

where

$$
\mathcal{L}=\left[\begin{array}{ccc}
1 & \cdots & 1 \\
e^{-j 2 \pi(\mathrm{Q} / 2)(1 / K)} & \cdots & e^{j 2 \pi(\mathrm{Q} / 2)(1 / K)} \\
\vdots & & \vdots \\
e^{-j 2 \pi(\mathrm{Q} / 2)((N-1) / K)} & \cdots & e^{j 2 \pi(Q / 2)((N-1) / K)}
\end{array}\right] .
$$

The solution of (16) is given by

$$
\mathbf{h}_{l}=\mathscr{L}^{\dagger} \mathbf{g}_{l}
$$

In practice, only a few pilot symbols are available for channel estimation. From (8) the channel BEM coefficients can be obtained by solving the following LS problem (assuming that $x\left[n_{p}\right]=1$, for $p=0, \ldots, P-1$ )

$$
\min _{\mathbf{h}_{l}}\left\|\mathbf{y}_{t, l}-\tilde{\mathscr{L}}_{l} \mathbf{h}_{l}\right\|^{2}
$$

where

$$
\tilde{\mathscr{L}}_{l}=\left[\begin{array}{ccc}
e^{-j 2 \pi(Q / 2)\left(n_{0, l} / K\right)} & \cdots & e^{j 2 \pi(Q / 2)\left(n_{0, l} / K\right)} \\
\vdots & & \vdots \\
e^{-j 2 \pi(Q / 2)\left(n_{P-1, l} / K\right)} & \cdots & e^{j 2 \pi(Q / 2)\left(n_{P-1, l} / K\right)}
\end{array}\right] .
$$

The solution of (19) is obtained by

$$
\mathbf{h}_{l}=\tilde{\mathscr{L}}_{l}^{\dagger} \mathbf{y}_{t, l} .
$$

It has been shown in [6] that when critically sampling the Doppler spectrum $(K=N)$ and ignoring the modeling error, the optimal training strategy consists of inserting equipowered, equispaced pilot symbols. However, critically sampling the Doppler spectrum results in an error floor due to the large modeling error. On the other hand, oversampling the Doppler spectrum $(K=r N$, with integer $r>1)$ reduces the modeling error when the ideal case is considered $[3,26,27]$, that is, when (16) is applied. However, this channel estimate is sensitive to noise when PSAM channel estimation is used.

A robust channel estimate can then be obtained by combining the optimal-MMSE-interpolation-based channel estimate obtained in (9) with the BEM channel estimate obtained in (16) as follows.

(i) First, obtain the channel estimate $\hat{\mathbf{g}}_{l}$ as in (9).

(ii) Second, obtain the LS solution of the following problem:

$$
\min _{\mathbf{h}_{l}}\left\|\hat{\mathbf{g}}_{l}-\mathcal{L} \mathbf{h}_{l}\right\|^{2} .
$$

The solution of (22) can be obtained as

$$
\mathbf{h}_{l}=\mathcal{L}^{\dagger} \hat{\mathbf{g}}_{l},
$$

or equivalently in one step as

$$
\mathbf{h}_{l}=\mathcal{L}^{\dagger} \mathbf{W}_{\mathrm{MMSE}, l}^{H} \mathbf{y}_{t, l} .
$$

Even though this applies to critically sampled Doppler spectrum as well as to oversampled Doppler spectrum, little gain is obtained when combining the MMSE-interpolationbased channel estimate with the critically sampled BEM ( $K=$ $N$ ), as will be clear in Section 5 . 


\subsection{PSAM direct equalization}

In this section we propose a PSAM-based direct equalization of doubly selective channels, where the time-varying FIR equalizer coefficients are obtained directly without passing through the channel estimation step. Applying the timevarying FIR equalizer $w^{(r)}[n ; \nu]$ to the $r$ th receive antenna sequence $y^{(r)}[n]$, an estimate of $x[n]$ (within a specific range as indicated later on) can be obtained as

$$
\hat{x}[n-d]=\sum_{r=1}^{N_{r}} \sum_{\nu=-\infty}^{\infty} w^{(r)}[n ; \nu] y^{(r)}[n-\nu],
$$

where $d$ is the decision delay.

Using the BEM to design the time-varying FIR filters, each time-varying FIR equalizer $w^{(r)}[n ; \nu]$ is designed to have $L^{\prime}+1$ taps. The time-variation of each tap is modeled by $Q^{\prime}+1$ complex exponential basis functions with frequencies on some DFT grid not necessarily the same DFT grid as the one for the channel. Therefore, the time-varying FIR filter corresponding to the $r$ th receive antenna can be written as

$$
w^{(r)}[n ; \nu]=\sum_{l^{\prime}=0}^{L^{\prime}} \delta\left[\nu-l^{\prime}\right] \sum_{q^{\prime}=-Q^{\prime} / 2}^{Q^{\prime} / 2} w_{q^{\prime}, l^{\prime}}^{(r)} e^{j 2 \pi q^{\prime} n / K},
$$

where $w_{q^{\prime}, l^{\prime}}^{(r)}$ is the BEM coefficient of the $q^{\prime}$ th basis of the $l^{\prime}$ th tap of the equalizer, and $K$ is the BEM resolution of the equalizer. Substituting (26) in (25) we obtain

$$
\hat{x}[n-d]=\sum_{l^{\prime}=0}^{L^{\prime}} \sum_{q^{\prime}=-Q^{\prime} / 2}^{Q^{\prime} / 2} e^{j 2 \pi q^{\prime} n / K} w_{q^{\prime}, l^{\prime}}^{(r)} y^{(r)}\left[n-l^{\prime}\right] .
$$

Define $\mathbf{w}^{(r)}=\left[\mathbf{w}_{-Q^{\prime} / 2}^{(r) T}, \ldots, \mathbf{w}_{Q^{\prime} / 2}^{(r) T}\right]^{T}$ with $\mathbf{w}_{q^{\prime}}^{(r)}=\left[w_{q^{\prime}, 0}^{(r)}, \ldots\right.$, $\left.w_{q^{\prime}, L^{\prime}}^{(r)}\right]^{T}$, then a block level formulation of (27) can be written as

$$
\hat{\mathbf{x}}_{*}^{T}=\sum_{r=1}^{N_{r}} \mathbf{w}^{(r) T} y^{(r)}=\mathbf{w}^{T} y
$$

where $\hat{\mathbf{x}}_{*}=\left[\hat{x}\left[L^{\prime}-d\right], \ldots, \hat{x}[N-d-1]\right]^{T}, \mathbf{w}=\left[\mathbf{w}^{(1) T}, \ldots\right.$, $\left.\mathbf{w}^{\left(N_{r}\right) T}\right]^{T}$, and $y=\left[\mathcal{y}^{(1) T}, \ldots, y^{\left(N_{r}\right) T}\right]^{T}$, with $y^{(r)}$ a $\left(Q^{\prime}+1\right)\left(L^{\prime}+1\right) \times\left(N-L^{\prime}\right)$ matrix containing the timeand frequency-shifts of the received sequence given by $y^{(r)}=\left[\mathbf{y}_{-Q^{\prime} / 2,0}^{(r)}, \ldots, \mathbf{y}_{-Q^{\prime} / 2, L^{\prime}}^{(r)}, \ldots, \mathbf{y}_{Q^{\prime} / 2, L^{\prime}}^{(r)}, \ldots, \mathbf{y}_{Q^{\prime} / 2, L^{\prime}}^{(r)}\right]^{T}$. The $q^{\prime}$ th frequency-shifted and $l^{\prime}$ th time-shifted version of the received sequence on the $r$ th receive antenna is given by

$$
\mathbf{y}_{q^{\prime}, l^{\prime}}^{(r)}=\mathbf{D}_{q^{\prime}} \mathbf{Z}_{l^{\prime}} \mathbf{y}^{(r)}
$$

with $\mathbf{Z}_{l^{\prime}}$ and $\mathbf{D}_{q^{\prime}}$ defined as

$$
\begin{aligned}
& \mathbf{Z}_{l^{\prime}}=\left[\mathbf{0}_{\left(N-L^{\prime}\right) \times\left(L^{\prime}-l^{\prime}\right)}, \mathbf{I}_{N-L^{\prime}}, \mathbf{0}_{\left(N-L^{\prime}\right) \times l^{\prime}}\right], \\
& \mathbf{D}_{q^{\prime}}=\operatorname{diag}\left\{\left[1, \ldots, e^{j 2 \pi q^{\prime}\left(N-L^{\prime}-1\right) / K}\right]^{T}\right\},
\end{aligned}
$$

and $\mathbf{y}^{(r)}=\left[y^{(r)}[0], \ldots, y^{(r)}[N-1]\right]^{T}$.
Assume that we have $P$ pilot symbols collected in the vector $\mathbf{x}_{t}=\left[x\left[n_{0}\right], \ldots, x\left[n_{P-1}\right]\right]^{T}$. Note that for direct equalization, the optimal training strategy is unknown. Therefore, we assume that the pilot symbols are inserted at positions $n_{0}, \ldots, n_{P-1}$ and that the pilot symbols are not necessarily surrounded with zeros on each side. Defining $y_{t}$ as the collection of columns of $y$ that corresponds to the training symbol positions subject to some decision delay, defining $[\mathcal{Y}]_{i}$ as the $i$ th column of the matrix $\mathcal{Y}$, and defining $y_{t}=\left[[y]_{d+n_{0}}, \ldots,[y]_{d+n_{P-1}}\right]$, the PSAM direct equalizer BEM coefficients are generally obtained by minimizing the following cost function:

$$
\min _{\mathbf{w}}\left\|\mathbf{w}^{T} y_{t}-\mathbf{x}_{t}^{T}\right\|^{2}
$$

which is obtained as

$$
\mathbf{w}=\left(y_{t}^{*} y_{t}^{T}\right)^{-1} y_{t}^{*} \mathbf{x}_{t}
$$

The solution in (32) is no more than the LS solution. A more robust LS solution can be obtained by solving the regularized LS problem as [28]

$$
\min _{\mathbf{w}}\left\|\mathbf{w}^{T} y_{t}-\mathbf{x}_{t}^{T}\right\|^{2}+\left\|\mathbf{R}_{\widetilde{v}}^{1 / 2} \mathbf{w}\right\|^{2}
$$

The solution of this problem is then obtained as

$$
\mathbf{w}=\left(y_{t}^{*} y_{t}^{T}+\mathbf{R}_{\tilde{v}}\right)^{-1} y_{t}^{*} \mathbf{x}_{t}
$$

which reduces to

$$
\mathbf{w}=\left(y_{t}^{*} y_{t}^{T}+\sigma_{n}^{2} \mathbf{I}_{P}\right)^{-1} y_{t}^{*} \mathbf{x}_{t}
$$

for the additive white Gaussian noise $\mathbf{R}_{\tilde{v}}=\sigma_{n}^{2} \mathbf{I}$.

A ZF time-varying FIR equalizer can be obtained as in (32) if the number of training symbols $P \geq N_{r}\left(Q^{\prime}+1\right)\left(L^{\prime}+1\right)$. This is achieved provided that $N_{r}\left(Q^{\prime}+1\right)\left(L^{\prime}+1\right) \geq(Q+$ $\left.Q^{\prime}+1\right)\left(L+L^{\prime}+1\right)($ see $[1])$. This is a necessary condition for the channel matrix $\mathscr{H}$ (see $(40)$ ) to be of full column rank, and therefore for a ZF time-varying FIR serial linear equalizer (SLE) to exist. Note that for (35), this condition is relaxed.

\section{BLIND AND SEMIBLIND TECHNIQUES}

\subsection{Channel estimation}

In this section we focus again on the problem of channel estimation, where the channel estimate is obtained via blind techniques or semiblind techniques. We first discuss deterministic blind channel estimation procedure. In blind methods the channel is estimated up to a scalar ambiguity and, for example, computed from the singular value decomposition (eigenvalue decomposition) of a large matrix. To resolve the scalar ambiguity, a blind technique combined with a training-based technique is favorable resulting in a semiblind technique, which is discussed in a second section. 


\subsubsection{Blind channel estimation}

Here we discuss a deterministic subspace based blind channel estimation [29]. It operates on time- and frequency-shifted versions of the received sequence. Assume that $\left(Q^{\prime}+1\right)$ frequency-shifts and $\left(L^{\prime}+1\right)$ time-shifts of the received sequence related to the $r$ th receive antenna are stored in a $\left(Q^{\prime}+1\right)\left(L^{\prime}+1\right) \times\left(N-L^{\prime}\right)$ matrix $y^{(r)}$.

Approximating the doubly selective channel using the $\mathrm{BEM}$, we can write the received vector at the $r$ th receive antenna $\mathbf{y}^{(r)}=\left[y^{(r)}[0], \ldots, y^{(r)}[N-1]\right]^{T}$ as

$$
\mathbf{y}^{(r)}=\sum_{l=0}^{L} \sum_{q=-Q / 2}^{Q / 2} h_{q, l}^{(r)} \overline{\mathbf{D}}_{q} \overline{\mathbf{Z}}_{l} \mathbf{x}+\mathbf{v}^{(r)}
$$

where $\overline{\mathbf{D}}_{q}=\operatorname{diag}\left\{\left[1, \ldots, e^{j 2 \pi q(N-1) / K}\right]^{T}\right\}, \overline{\mathbf{Z}}_{l}=\left[\mathbf{0}_{N \times(L-l)}, \mathbf{I}_{N}\right.$, $\left.\mathbf{0}_{N \times l}\right], \mathbf{x}=[x[-L], \ldots, x[N-1]]^{T}$, and $\mathbf{v}^{(r)}$ is defined similar to $\mathbf{y}^{(r)}$. Hence, $\mathbf{y}_{q^{\prime}, l^{\prime}}^{(r)}$ can be written as

$$
\mathbf{y}_{q^{\prime}, l^{\prime}}^{(r)}=\sum_{l=0}^{L} \sum_{q=-Q / 2}^{Q / 2} e^{j 2 \pi q\left(L^{\prime}-l^{\prime}\right) / K} h_{q, l}^{(r)} \mathbf{D}_{q+q^{\prime}} \widetilde{\mathbf{Z}}_{l+l^{\prime}} \mathbf{x}+\mathbf{v}_{q^{\prime}, l^{\prime}}^{(r)},
$$

where $\widetilde{\mathbf{Z}}_{k}=\left[\mathbf{0}_{\left(N-L^{\prime}\right) \times\left(L+L^{\prime}-k\right)}, \mathbf{I}_{N-L^{\prime}}, \mathbf{0}_{\left(N-L^{\prime}\right) \times k}\right]$, and $\mathbf{v}_{q^{\prime}, l^{\prime}}^{(r)}$ is similarly defined as $\mathbf{y}_{q^{\prime}, l^{\prime}}^{(r)}$.

Define $\mathcal{X}=\left[\mathbf{x}_{-\left(Q^{\prime}+Q\right) / 2,0}, \ldots, \mathbf{x}_{-\left(Q^{\prime}+Q\right) / 2,\left(L+L^{\prime}+1\right)}, \ldots\right.$, $\left.\mathbf{x}_{\left(Q^{\prime}+Q\right) / 2,\left(L+L^{\prime}+1\right)}\right]^{T}$ where $\mathbf{x}_{p, k}$ is the $p$ th frequency-shifted and $k$ th time-shifted version of the transmitted sequence obtained as

$$
\mathbf{x}_{p, k}=\mathbf{D}_{p} \widetilde{\mathbf{Z}}_{k} \mathbf{x}
$$

A relationship between $\mathcal{y}^{(r)}$ and the transmitted sequence can be obtained by substituting (36) in $y^{(r)}$ resulting in

$$
\mathcal{y}^{(r)}=\mathscr{H}^{(r)} \mathcal{X}+\mathcal{V}^{(r)}
$$

where $\mathscr{H}^{(r)}$ is a $\left(Q^{\prime}+1\right)\left(L^{\prime}+1\right) \times\left(Q+Q^{\prime}+1\right)\left(L+L^{\prime}+1\right)$ matrix given by

$$
\begin{aligned}
& \mathscr{H}^{(r)} \\
& =\left[\begin{array}{cccc}
\boldsymbol{\Omega}_{-Q / 2} \mathscr{H}_{-Q / 2}^{(r)} & \ldots & \boldsymbol{\Omega}_{\mathrm{Q} / 2} \mathscr{H}_{\mathrm{Q} / 2}^{(r)} & 0 \\
\ddots & & \ddots & \\
\mathbf{0} & \boldsymbol{\Omega}_{-Q / 2} \mathcal{H}_{-Q / 2}^{(r)} & \cdots & \boldsymbol{\Omega}_{\mathrm{Q} / 2} \mathscr{H}_{\mathrm{Q} / 2}^{(r)}
\end{array}\right],
\end{aligned}
$$

where $\boldsymbol{\Omega}_{q}=\operatorname{diag}\left\{\left[e^{-j 2 \pi q L^{\prime} / K}, \ldots, 1\right]^{T}\right\}$, and $\mathscr{H}_{q^{(r)}}^{(s)}$ given by

$$
\mathscr{H}_{q}^{(r)}=\left[\begin{array}{ccccc}
h_{q, 0}^{(r)} & \cdots & h_{q, L}^{(r)} & & 0 \\
& \ddots & & \ddots & \\
& & h_{q, 0}^{(r)} & \cdots & h_{q, L}^{(r)}
\end{array}\right] .
$$

The noise matrix $\mathcal{V}^{(r)}$ is similarly defined as $\mathcal{y}^{(r)}$.

Stacking the $N_{r}$ resulting matrices $y=\left[\mathcal{y}^{(1) T}, \ldots\right.$, $\left.y^{\left(N_{r}\right) T}\right]^{T}$, we obtain

$$
y=\mathscr{H} X+\mathcal{V}
$$

where $\mathscr{H}=\left[\mathscr{H}^{(1) T}, \ldots, \mathscr{H}^{\left(N_{r}\right) T}\right]^{T}$ and $\mathcal{V}=\left[\mathcal{V}^{(1) T}, \ldots\right.$, $\left.\mathcal{V}^{\left(N_{r}\right) T}\right]^{T}$.

Let us assume the following.

(A1) $\mathscr{H}$ has full column $\operatorname{rank}\left(Q+Q^{\prime}+1\right)\left(L+L^{\prime}+1\right)$ (see [1]).

(A2) $\mathcal{X}$ has full row $\operatorname{rank}\left(Q+Q^{\prime}+1\right)\left(L+L^{\prime}+1\right)[9]$.

(A3) $N-L^{\prime} \geq N_{r}\left(Q^{\prime}+1\right)\left(L^{\prime}+1\right)$.

Under these assumptions, the matrix $y$ has $I=N_{r}\left(Q^{\prime}+\right.$ 1) $\left(L^{\prime}+1\right)-\left(Q+Q^{\prime}+1\right)\left(L+L^{\prime}+1\right)$ zero singular values in the noiseless case (in the noisy case, these singular vectors are referred to as noise singular values associated with the $I$ minimum singular vectors, see below). Suppose that $\mathbf{u}_{1}, \ldots, \mathbf{u}_{I}$ are the $I$ left singular vectors corresponding to the $I$ zero singular values. Then we can write

$$
\mathbf{u}_{i}^{H} \mathscr{H}=\mathbf{0}_{1 \times\left(Q+Q^{\prime}+1\right)\left(L+L^{\prime}+1\right)}, \quad \forall i \in\{1, \ldots, I\} .
$$

Define $\mathbf{u}_{i}=\left[\mathbf{u}_{i}^{(1) T}, \ldots, \mathbf{u}_{i}^{\left(N_{r}\right) T}\right]^{T}, \mathbf{u}_{i}^{(r)}=\left[\mathbf{u}_{i,-Q^{\prime} / 2}^{(r) T}, \ldots, \mathbf{u}_{i, Q^{\prime} / 2}^{(r) T}\right]^{T}$, and $\mathbf{u}_{i, q^{\prime}}^{(r)}=\left[u_{i, q^{\prime}, 0}^{(r)}, \ldots, u_{i, q^{\prime}, L^{\prime}}^{(r)}\right]^{T}$. Then (43) can be equivalently written as

$$
u_{i}^{H} \mathbf{h}=\mathbf{0}_{1 \times\left(Q+Q^{\prime}+1\right)\left(L+L^{\prime}+1\right)}, \quad \forall i \in\{1, \ldots, I\},
$$

where $\mathbf{h}=\left[\mathbf{h}^{(1) T}, \ldots, \mathbf{h}^{\left(N_{r}\right) T}\right]^{T}$ with $\mathbf{h}^{(r)}=\left[\mathbf{h}_{-Q / 2}^{(r) T}, \ldots, \mathbf{h}_{Q / 2}^{(r) T}\right]^{T}$, and $\mathbf{h}_{q}^{(r)}=\left[h_{q, 0}^{(r)}, \ldots, h_{q, L}^{(r)}\right]^{T}$. In $(44), \boldsymbol{U}_{i}=\left[\boldsymbol{U}_{i}^{(1)^{T}}, \ldots, \boldsymbol{U}_{i}^{\left(N_{r}\right) T}\right]^{T}$, where $\mathcal{U}_{i}^{(r)}$ is defined as

$$
u_{i}^{(r)}=\left[\begin{array}{ccccc}
\boldsymbol{\Omega}_{1}^{-Q / 2} u_{i,-Q^{\prime} / 2}^{(r)} \boldsymbol{\Omega}_{2}^{\mathrm{Q} / 2} & \cdots & \boldsymbol{\Omega}_{1}^{-Q / 2} u_{i, Q^{\prime} / 2}^{(r)} \boldsymbol{\Omega}_{2}^{\mathrm{Q} / 2} & & \mathbf{0} \\
& \ddots & & \ddots & \\
\mathbf{0} & & \boldsymbol{\Omega}_{1}^{\mathrm{Q} / 2} u_{i,-Q^{\prime} / 2}^{(r)} \boldsymbol{\Omega}_{2}^{-Q / 2} & \cdots & \boldsymbol{\Omega}_{1}^{\mathrm{Q} / 2} u_{i, Q^{\prime} / 2}^{(r)} \boldsymbol{\Omega}_{2}^{-Q / 2}
\end{array}\right]
$$


with $u_{i, q^{\prime}}^{(r)}$ an $(L+1) \times\left(L^{\prime}+L+1\right)$ Toeplitz matrix given by

$$
u_{i, q^{\prime}}^{(r)}=\left[\begin{array}{ccccc}
u_{i, q^{\prime}, 0}^{(r)} & \cdots & u_{i, q^{\prime}, L^{\prime}}^{(r)} & & 0 \\
& \ddots & & \ddots & \\
0 & & u_{i, q^{\prime}, 0}^{(r)} & \cdots & u_{i, q^{\prime}, L^{\prime}}^{(r)}
\end{array}\right],
$$

$\boldsymbol{\Omega}_{1}=\operatorname{diag}\left\{\left[1, e^{j 2 \pi / K}, \ldots, e^{j 2 \pi L / K}\right]^{T}\right\}$, and $\boldsymbol{\Omega}_{2}=\operatorname{diag}\{[1$, $\left.\left.e^{j 2 \pi / K}, \ldots, e^{j 2 \pi\left(L+L^{\prime}\right) / K}\right]^{T}\right\}$.

Collecting the results for the $I$ left singular vectors we obtain

$$
u^{H} \mathbf{h}=\mathbf{0}_{I\left(Q+Q^{\prime}+1\right)\left(L+L^{\prime}+1\right) \times 1},
$$

where $\mathcal{U}=\left[U_{1}, \ldots, \mathcal{U}_{I}\right]$, from which $\mathbf{h}$ can be computed up to a scalar ambiguity. In the presence of noise, we compute the $I$ left singular vectors of $\mathcal{y}$ corresponding to the $I$ smallest singular values. We denote these vectors as $\widehat{\mathbf{u}}_{1}, \ldots, \widehat{\mathbf{u}}_{I}$, and obtain the corresponding $\widehat{u}$ in a similar fashion as $u$. The channel estimate is then obtained as

$$
\min _{\mathbf{h}}\left\|\widehat{u}^{H} \mathbf{h}\right\|^{2} .
$$

The solution is obtained by the singular vector of $\widehat{u}$ corresponding to the smallest singular value.

\subsubsection{Semiblind channel estimation}

In blind methods, the channel is estimated up to a scalar multiplication. To resolve the scalar ambiguity, training symbols are used along with the blind technique resulting in the so-called semiblind technique. In semiblind techniques, the channel estimate is obtained by minimizing a cost function consisting of two parts. The first part corresponds to the training, and the second part corresponds to the blind estimation.

First, let us consider the channel estimate that relies on known symbols. To facilitate channel estimation, we write the input-output relationship as

$$
\mathbf{y}^{T}=\mathbf{h}^{T}\left(\mathbf{I}_{N_{r}} \otimes \mathcal{X}_{\mathrm{sb}}\right)+\mathbf{v}^{T},
$$

where $\mathbf{y}=\left[\mathbf{y}^{(1) T}, \ldots, \mathbf{y}^{\left(N_{r}\right) T}\right]^{T}, \mathbf{v}=\left[\mathbf{v}^{(1) T}, \ldots, \mathbf{v}^{\left(N_{r}\right) T}\right]^{T}$, and the $(Q+1)(L+1) \times N$ matrix $\mathcal{X}_{\mathrm{sb}}=\left[\overline{\mathbf{x}}_{-Q / 2,0}, \ldots, \overline{\mathbf{x}}_{Q / 2, L}\right]^{T}$ with the $q$ th frequency-shift and $l$ th time-shift of the transmitted sequence $\mathbf{x}$ is given by

$$
\overline{\mathbf{x}}_{q, l}=\overline{\mathbf{D}}_{q} \overline{\mathbf{Z}}_{l} \mathbf{x}
$$

Let us assume that $N_{t}$ symbols are used for training, and the remaining symbols are data symbols. Collecting the received symbols that correspond to training in one vector $\mathbf{y}_{t}$, and the corresponding columns of $\mathcal{X}_{\mathrm{sb}}$ in a matrix $\mathcal{X}_{\mathrm{sb}, t}$, we can write the received sequence corresponding to training as

$$
\mathbf{y}_{t}=\left(\mathbf{I}_{N_{r}} \otimes \mathcal{X}_{\mathrm{sb}, t}^{T}\right) \mathbf{h}+\mathbf{v}_{t} .
$$

An LS channel estimate $\hat{\mathbf{h}}_{\mathrm{tr}}$ is then computed based on the training symbols as

$$
\widehat{\mathbf{h}}_{\mathrm{tr}}=\left(\mathbf{I}_{N_{r}} \otimes \mathcal{X}_{\mathrm{sb}, t}^{T}\right)^{\dagger} \mathbf{y}_{t} .
$$

To avoid the under-determined case, that is, the matrix $\mathbf{I}_{N_{r}} \otimes$ $X_{\mathrm{sb}, t}^{T}$ is not of full column rank, it is required that the number of training symbols be $N_{t} \geq(Q+1)(L+1)$. To have nonoverlapping data and training the optimal training strategy again consists of $(Q+1)$ clusters of $2 L+1$ training symbols. Each cluster consists of a training symbol and $L$ surrounding zeros on each side [6]. Therefore, the training overhead is actually $(Q+1)(2 L+1)$, and the non-overlapping part is $N_{t}=$ $(Q+1)(L+1)$. This training overhead can be greatly reduced by combining the training with a blind estimation technique resulting in a semiblind technique.

The semiblind channel estimate can be obtained as

$$
\widehat{\mathbf{h}}_{\mathrm{sb}}=\arg \min _{\mathbf{h}}\left\{\alpha \mathbf{h}^{T} \widehat{u}^{*} \widehat{u}^{T} \mathbf{h}^{*}+\left\|\mathbf{y}_{t}^{T}-\mathbf{h}^{T}\left(\mathbf{I}_{N_{r}} \otimes \mathcal{X}_{\mathrm{sb}, t}\right)\right\|^{2}\right\},
$$

where $\alpha>0$ is a weighting factor. In (53) the first part corresponds to blind estimation while the second part corresponds to training. If $\alpha$ is large, then the blind method is emphasized, whereas the LS training-based estimation is emphasized for small $\alpha$.

The solution for the semiblind channel estimation problem is then obtained as

$$
\hat{\mathbf{h}}_{\mathrm{sb}}=\left(\alpha \widehat{\mathcal{u}} \widehat{u}^{H}+\mathbf{I}_{N_{r}} \otimes\left(\mathcal{X}_{\mathrm{sb}, t} \mathcal{X}_{\mathrm{sb}, t}^{H}\right)^{T}\right)^{-1}\left(\mathbf{I}_{N_{r}} \otimes \mathcal{X}_{\mathrm{sb}, t}^{H}\right)^{T} \mathbf{y}_{t} .
$$

\subsection{Direct equalization}

In direct equalization the equalizer coefficients are obtained directly without passing through the channel estimation stage. There are many techniques that can be applied to obtain directly the equalizer coefficients for the case of frequency-selective channels. These techniques are either stochastic or deterministic. However, due to the fact that we assume the BEM channel model, and the fact that the channel BEM coefficients may change from block to block, stochastic techniques cannot be applied. In this section we will rely on deterministic direct equalization techniques. We first discuss a deterministic blind direct equalization technique that relies on the so-called mutually referenced equalization (MRE). MRE has been successfully applied to TI channels $[8,9]$. In MRE the idea is to tune a number of equalizers, where the output of one of these tuned equalizers is used to train the other equalizers in a mutual fashion. For the case of time-varying channels, the same idea can be applied, but taking into account the time- and the frequency-shifts of the received signal. A semiblind algorithm is again obtained by combining the training-based LS method and the blind MRE method.

\subsubsection{Blind direct equalization}

The idea of MRE-based blind direct equalization is to tune various equalizers associated with reconstructing the transmitted signal subject to a time- and frequency-shift. Define 
$\mathbf{w}_{p, k}^{T}$ as the time-varying FIR equalizer that reconstructs the $p$ th frequency-shifted and $k$ th time-shifted (delayed) version of the received sequence in the noiseless case as

$$
\mathbf{w}_{p, k}^{T} y=\mathbf{x}^{T} \tilde{\mathbf{Z}}_{k}^{T} \mathbf{D}_{p}
$$

In order to have mutually referenced equalizers training each other for frequency-shifts $p \in\left\{-\left(Q+Q^{\prime}\right) / 2, \ldots,\left(Q+Q^{\prime}\right) / 2\right\}$ and time-shifts (delays) $k \in\left\{0, \ldots, L+L^{\prime}\right\}$, we set $\mathbf{x}=$ $\left[\mathbf{0}_{1 \times\left(L+L^{\prime}\right)}, \mathbf{x}_{*}^{T}, \mathbf{0}_{1 \times\left(L+L^{\prime}\right)}\right]^{T}$, with $\mathbf{x}_{*}$ a data vector of length $M=$ $N-L-2 L^{\prime}$.

Define $\mathcal{y}_{p, k}=\mathcal{y}_{-p} \breve{\mathbf{Z}}_{k}$, with $\breve{\mathbf{Z}}_{k}=\left[\mathbf{0}_{M \times k}, \mathbf{I}_{M}, \mathbf{0}_{M \times\left(L+L^{\prime}-k\right)}\right]^{T}$. Hence, we can write (55) as

$$
\mathbf{w}_{p, k}^{T} y_{p, k}=\mathbf{x}_{*}^{T}
$$

In order for (56) to lead to a ZF solution in the noiseless case, we require that assumptions (A1) and (A2) required for channel estimation to be satisfied in addition to

(A3') the data length $M>N_{r}\left(Q^{\prime}+1\right)\left(L^{\prime}+1\right)$,

Taking the 0th frequency-shift and the 0th time-shift equalizer $\mathbf{w}_{0,0}$ as a reference equalizer and collecting the different equalizer coefficients in one vector $\mathbf{w}=\left[\mathbf{w}_{0,0}^{T}\right.$, $\left.\mathbf{w}_{-\left(Q+Q^{\prime}\right) / 2,0}^{T}, \ldots, \ldots, \mathbf{w}_{-1, L+L^{\prime}}^{T}, \mathbf{w}_{0,1}^{T}, \ldots, \mathbf{w}_{\left(Q+Q^{\prime}\right) / 2, L+L^{\prime}}^{T}\right]^{T}$, we arrive at the following:

$$
\mathbf{w}^{T} \breve{y}=\mathbf{0}_{1 \times M\left(Q+Q^{\prime}+1\right)\left(L+L^{\prime}+1\right)},
$$

where

$$
\breve{y}=\left[\begin{array}{cccc}
y_{0,0} & y_{0,0} & \cdots & y_{0,0} \\
-y_{-\left(Q+Q^{\prime}\right) / 2,0} & 0 & & 0 \\
0 & -y_{-\left(Q+Q^{\prime}\right) / 2,1} & & \\
\vdots & & \ddots & \vdots \\
0 & \cdots & \mathbf{0} & -y_{\left(Q+Q^{\prime}\right) / 2, L+L^{\prime}}
\end{array}\right] .
$$

Note that in the noiseless case, it can be proven that the rank of $\breve{y}$ is $\left(Q+Q^{\prime}+1\right)^{2}\left(L+L^{\prime}+1\right)^{2}-1$.

The different $\mathbf{w}_{p, k}$ 's are linearly independent and cannot be obtained from each other. The different equalizers can be used as rows of a $\left(Q+Q^{\prime}+1\right)\left(L+L^{\prime}+1\right) \times N_{r}\left(Q^{\prime}+1\right)\left(L^{\prime}+1\right)$ matrix $\mathcal{W}$. Based on the ZF conditions we obtain the following relation:

$$
\mathcal{W} \mathscr{H}=\gamma \mathbf{I}_{\left(Q+Q^{\prime}+1\right)\left(L+L^{\prime}+1\right)},
$$

where $\gamma$ is some scalar ambiguity satisfying

$$
\mathbf{w}_{0,0}^{T} y_{0,0}=\mathbf{w}_{p, k}^{T} y_{p, k}=\gamma \mathbf{x}_{*}^{T}, \quad \forall p, k p \neq 0, k \neq 0 .
$$

We can solve (57) either by using LS or by a subspace decomposition [9]. For the LS solution we constrain the first entry of $\mathbf{w}$ to 1 and solve (57) for the remaining entries of $\overline{\mathbf{w}}$ resulting in

$$
\overline{\mathbf{w}}_{\mathrm{LS}}^{T}=\left(\overline{\breve{y}}^{H} \overline{\mathrm{y}}^{-1} \overline{\breve{y}}^{H} \overline{\mathbf{y}}\right.
$$

where $\overline{\mathscr{y}}$ is the matrix obtained after removing the first row of $\breve{y}$ and $\bar{y}$ is this row multiplied by -1 . The subspace approach is obtained by taking $\|\mathbf{w}\|^{2}=1$, and then $\mathbf{w}$ is found as the left singular vector corresponding to the minimum singular value of $\breve{y}$.

Note that if channel estimation is required, then using (59) the channel can be estimated subject to some scalar ambiguity.

\subsubsection{Semiblind direct equalization}

The MRE blind algorithm estimates the transmitted signal up to a scalar ambiguity $\gamma$ (see (60)). In addition, the blind MRE is very complex. These two difficulties with the blind MRE can be resolved by combining training with the blind MRE method resulting in a so-called semiblind direct equalization method. The proposed semiblind approach consists of a combination of the training-based least-squares (LS) method [30] and the blind MRE method [8, 9], both wellknown for frequency-selective channels, but here applied to doubly selective channels. Again we consider different SLEs that detect different time- and frequency-shifted versions of the transmitted sequence. While during training periods, the training symbols are used to train all equalizers, during data transmission periods, each equalizer output is used to train the other equalizers.

Starting from (56), we assume that $N_{t}$ symbols in $\mathbf{x}_{*}$ are training symbols and the remaining $N_{d}=M-N_{t}$ symbols in $\mathbf{x}_{*}$ are data symbols. Let us then collect the training symbols of $\mathbf{x}_{*}$ in $\mathbf{x}_{*, t}$ and the data symbols of $\mathbf{x}_{*}$ in $\mathbf{x}_{*, d}$. Let us further collect the corresponding columns of $\mathcal{Y}_{p, k}$ in $\mathcal{Y}_{p, k, t}$ and $\mathcal{y}_{p, k, d}$, respectively. Splitting (56) into its training part and data part and stacking the results for $p \in$ $\left\{-\left(Q+Q^{\prime}\right) / 2, \ldots,\left(Q+Q^{\prime}\right) / 2\right\}$ and $k \in\left\{0, \ldots, L+L^{\prime}\right\}$ we arrive at the following:

$$
\mathbf{w}^{T}\left[\underline{y_{t}}, \underline{y_{d}}\right]=\left[\mathbf{x}_{*, t}^{T} \underline{\mathbf{I}}_{N_{t}}, \mathbf{x}_{*, d}^{T} \underline{\mathbf{I}}_{N_{t}}\right],
$$

where $\underline{y}_{t}$ and $\underline{y}_{d}$ are defined as

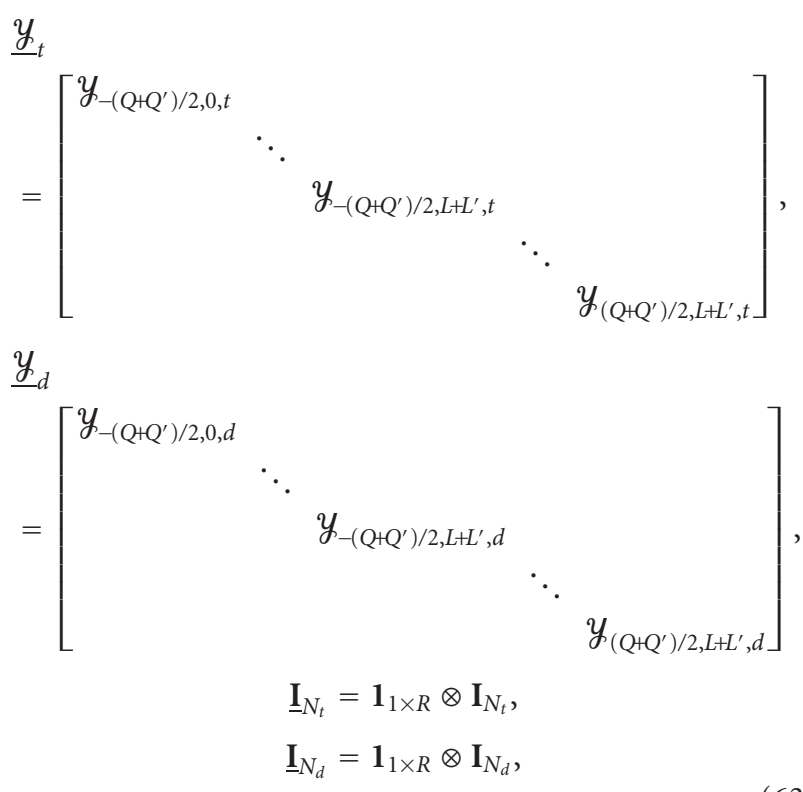

where $R=\left(Q+Q^{\prime}+1\right)\left(L+L^{\prime}+1\right)$. 
In the noisy case, we then have to solve

$$
\min _{\mathbf{w}, \mathbf{x}_{*, d}}\left\{\left\|\mathbf{w}^{T}\left[\underline{y_{t}}, \underline{y}_{d}\right]-\left[\mathbf{x}_{*, t}^{T} \underline{\mathbf{I}}_{N_{t}}, \mathbf{x}_{*, d}^{T} \underline{\mathbf{I}}_{N_{d}}\right]\right\|^{2}\right\}
$$

The solution for $\mathbf{x}_{*, d}$ is given by

$$
\hat{\mathbf{x}}_{*, d}^{T}=\mathbf{w}^{T} \underline{\underline{y}}{ }_{d} R^{-1} \underline{\mathbf{I}}_{N_{d}}^{T}
$$

Substituting (65) in (64), we obtain

$$
\min _{\mathbf{w}}\left\{\left\|\mathbf{w}^{T}\left[\underline{\underline{y}}, \underline{\underline{Z}}_{d}\right]-\left[\mathbf{x}_{*, t}^{T} \underline{\mathbf{I}}_{N_{t}}, \mathbf{0}_{1 \times N_{d} R}\right]\right\|^{2}\right\},
$$

where $\underline{Z}_{d}$ is given by

$$
\underline{\mathcal{Z}}_{d}\left[\begin{array}{ccc}
(R-1) \mathcal{y}_{-\left(Q+Q^{\prime}\right) / 2,0, d} & \cdots & -\mathcal{y}_{-\left(Q+Q^{\prime}\right) / 2,0, d} \\
\vdots & \ddots & \vdots \\
-\mathcal{y}_{\left(Q+Q^{\prime}\right) / 2, L+L^{\prime}, d} & \cdots & (R-1) \mathcal{y}_{\left(Q+Q^{\prime}\right) / 2, L+L^{\prime}, d}
\end{array}\right] .
$$

In (66), the left and right parts, respectively, correspond to the training-based LS method [30] and the blind MRE method $[8,9]$, now applied to doubly selective channels. So far in our analysis we considered all possible time- and frequency-shifts which means that the method exhibits a similar complexity as the blind technique. Due to the existence of the training part, we can limit the number of timeand frequency-shifts resulting in a much lower complexity semiblind technique. Therefore, we can redo the above analysis for time-shifts $k \in\left\{0, \ldots, K_{1}\right\}$ with $K_{1} \leq\left(L+L^{\prime}\right)$ and frequency-shifts $p \in\left\{-K_{2}, \ldots, K_{2}\right\}$ with $K_{2} \leq\left(Q+Q^{\prime}\right) / 2$. In other words, by the aid of training the number of tuned equalizers can be greatly reduced resulting in a much lower complexity than the blind techniques. In contrast, for blind techniques, for a $\mathrm{ZF}$ solution to be found, we require to tune the equalizers corresponding to all possible time- and frequency-shifts.

\section{SIMULATION RESULTS}

In this section, we evaluate the performance of the proposed channel estimation and direct equalization techniques. As direct techniques are still complex and prohibitive for practical reasons, only PSAM and semiblind techniques are simulated. We consider a rapidly time-varying channel simulated according to Jakes' model with $f_{\max }=100 \mathrm{~Hz}$, and sampling time $T=25 \mu \mathrm{s}$. The channel order is considered as $L=3$. The channel autocorrelation function is given by $r_{g, l}[k]=\sigma_{l}^{2} J_{0}\left(2 \pi f_{\max } k T\right)$, where $J_{0}$ is the zeroth-order Bessel function. In the simulations the channel is assumed to be WSS uncorrelated scattering with uniform power delay profile $\sigma_{l}^{2}=1$ for $l=0, \ldots, L$. For the simulations, we consider a window size of $N=800$ symbols unless stated otherwise. For the BEM, we consider the critically sampled Doppler spectrum $K=N$, as well as the oversampled Doppler spectrum with oversampling rate 2 (i.e., $K=2 N$ ). The number of basis functions is, therefore, chosen to be $Q=4$ for the critically sampled case, and $Q=8$ for the oversampled case.

\subsection{PSAM techniques}

\section{(i) PSAM-based channel estimation}

We use PSAM to estimate the channel. We consider equipowered and equispaced pilot symbols with $D$ the spacing between the pilots. The number of pilots is then computed as $P=\lfloor N / D\rfloor+1$. Since we adhere to the time-domain training [6], this training scheme consists of $P$-clusters, and each cluster consists of a training symbol and $L$ surrounding zeros at each side as explained in Figure 1. This means that the training overhead is $P(2 L+1) / N$.

First, we study the normalized channel MSE versus signal-to-noise ratio (SNR), where the MSE channel estimation is computed as

MSE

$$
=\frac{1}{N_{\mathrm{ch}} N_{r} N(L+1)} \sum_{i=1}^{N_{\mathrm{ch}}} \sum_{r=1}^{N_{r}} \sum_{n=0}^{N-1} \sum_{\nu=0}^{L}\left|\hat{h}^{(r)}[n ; \nu]-g^{(r)}[n ; \nu]\right|^{2},
$$

where $N_{\mathrm{ch}}$ is the number of channel realizations, and $\hat{h}^{(r)}[n ; \nu]$ is the estimate of (6) with the estimated BEM coefficients plugged in.

We evaluate the performance of the different estimation techniques, in particular, a BEM (21) with $K=N$, a combined BEM and MMSE (24) with $K=N$, a BEM with $K=2 N$, a combined BEM and MMSE with $K=2 N$, and finally the MMSE channel estimate (9). Note that the MMSE and BEM techniques will exactly coincide if and only if the underlying channel impulse response is perfectly described by the BEM. We consider the case when the spacing between pilot symbols is $D=165$ which corresponds to $P=5$ pilot symbols dedicated for channel estimation. This choice is well suited for $K=N$, where the number of BEM coefficients to be estimated is $Q+1=5$. We also consider the case when the spacing between pilot symbols is $D=95$, which corresponds to $P=9$ pilot symbols. This case is well suited for $K=2 \mathrm{~N}$ where 9 BEM coefficients are to be identified. As shown in Figure 2, when $D=165$ all the MSE channel estimates suffer from an early error floor. However, combining the critically sampled BEM with the MMSE results in a slightly better performance. On the other hand, when $D=95$ the performance of the BEM with $K=N$ suffers from an early error floor, which means that increasing the number of pilot symbols does not enhance the channel estimation technique. For the case when $K=2 N$, the MSE curves do not suffer from an early error floor. However, the oversampled BEM channel estimate is sensitive to noise. A significant improvement is obtained when the combined BEM and MMSE method is used, where a gain of $9 \mathrm{~dB}$ at MSE $=-20 \mathrm{~dB}$ is obtained over the conventional BEM method, when the oversampling rate is 2. Note also that the performance of the combined BEM and MMSE method when $K=2 N$ coincides with the performance of the MMSE only.

Second, we measure the MSE of the channel estimation techniques as a function of the maximum Doppler frequency. We design the system to have a maximum target Doppler frequency of $f_{\max }=100 \mathrm{~Hz}$ (used to design $\mathbf{W}_{\text {MMSE }}$ ). We then 


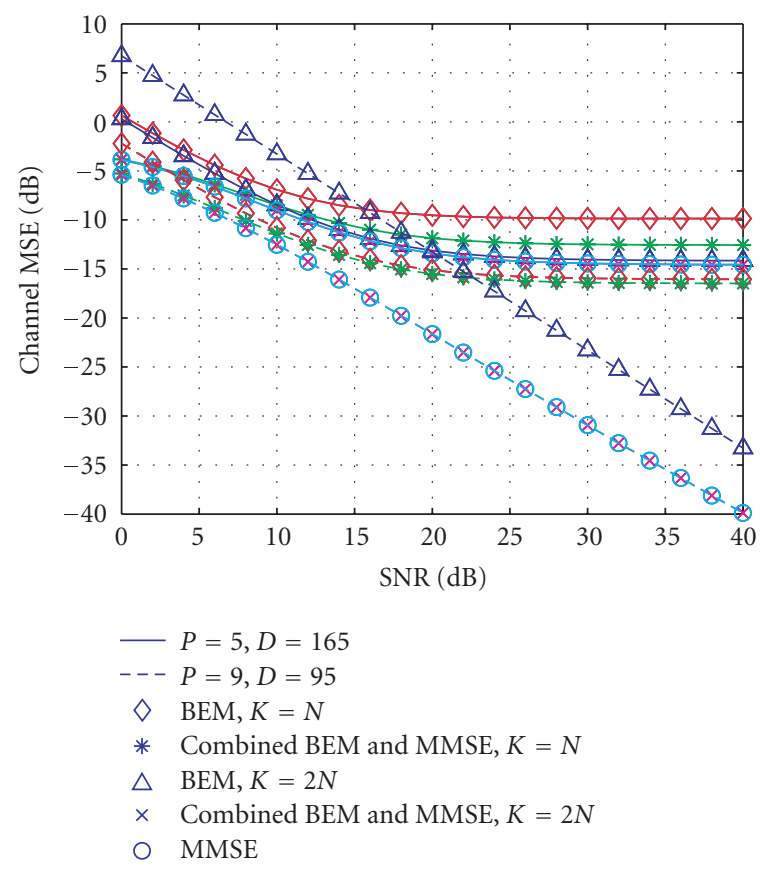

Figure 2: MSE versus SNR for $D=165$ and $D=95$.
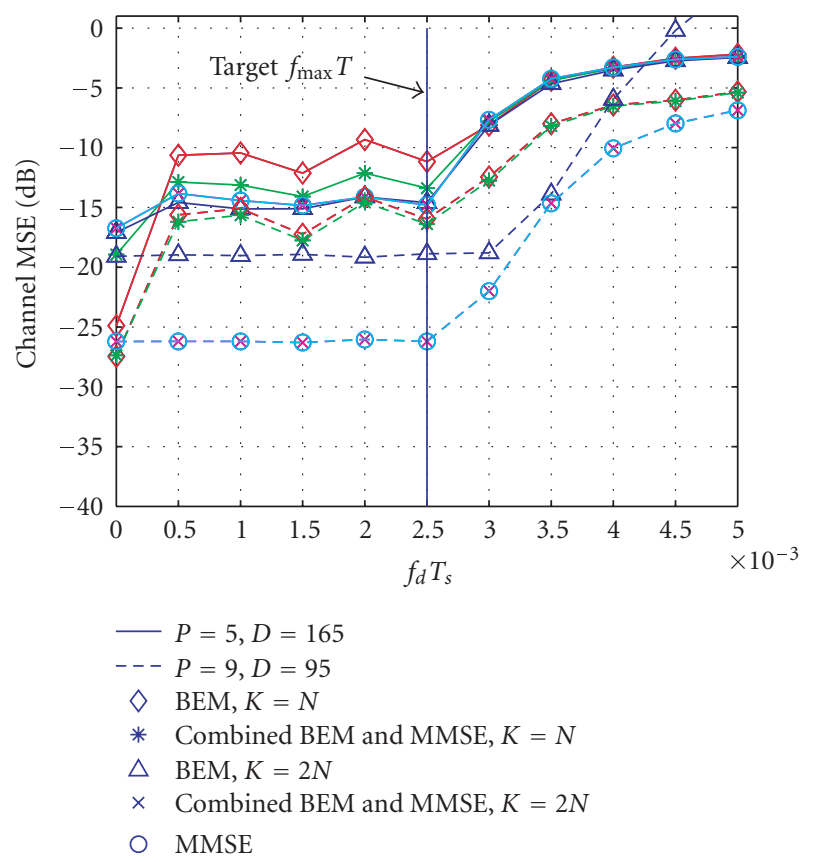

Figure 3: MSE versus $f_{\max }$ for $P=5, D=160$, and SNR $=25 \mathrm{~dB}$.

examine the performance of the channel estimation techniques for different maximum Doppler frequencies at a fixed $\mathrm{SNR}=25 \mathrm{~dB}$. The results are shown in Figure 3 for the case when $P=5$ pilot symbols are used for channel estimation, and when $P=9$ pilot symbols are used. For either case, the channel estimation techniques maintain a low MSE as long as the channel maximum Doppler frequency is smaller than the target maximum Doppler frequency.

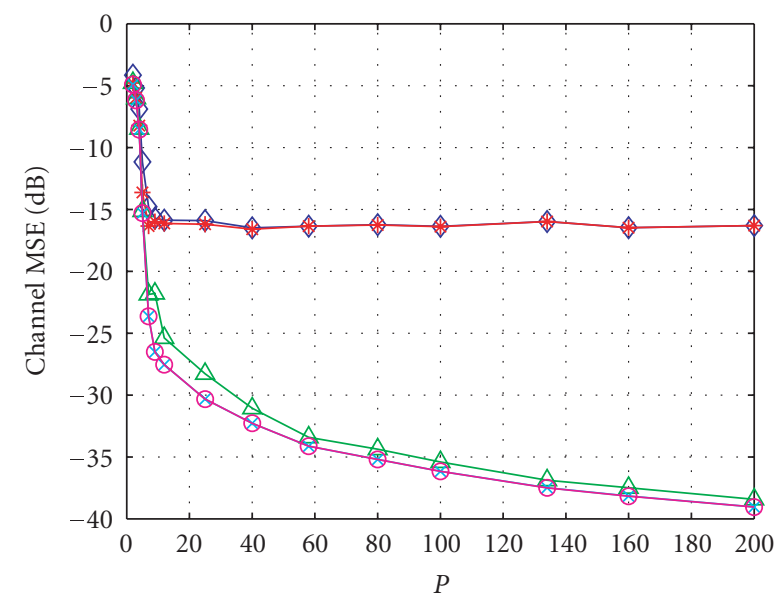

$$
\begin{aligned}
& \smile \text { BEM, } K=N \\
& * \text { Combined BEM and MMSE, } K=N \\
& \triangle \text { BEM, } K=2 N \\
& -\quad \text { Combined BEM and MMSE, } K=2 N \\
& - \text { MMSE }
\end{aligned}
$$

FIGURE 4: MSE channel estimation versus number of pilot symbols $P$ at $\mathrm{SNR}=25 \mathrm{~dB}$.

Third, we measure the MSE of the channel estimation techniques as a function of the number of pilot symbols $P$ (this can be easily translated to pilot spacing $D$ ). In this sense, we vary the number of pilot symbols $P$, while keeping the same maximum Doppler frequency $f_{\max }$ at $100 \mathrm{~Hz}$, and assuming the $\mathrm{SNR}=25 \mathrm{~dB}$. As shown in Figure 4 , for the case of $K=N$, increasing the number of pilot symbols (reducing $D)$ does not have a real impact on the MSE performance. This is not due to the choice of $D$, but rather due to the modeling error. On the other hand, the MSE channel estimation is significantly reduced by increasing the number of pilot symbols for $K=2 N$.

Finally, the estimated channel BEM coefficients are used to design time-varying FIR equalizers serial and decision feedback. We consider here a single-input multiple-output (SIMO) system with $N_{r}=2$ receive antennas. We consider the MMSE-SLE [1] as well as the MMSE serial decision feedback equalizer (MMSE-SDFE) [2]. For the case of the MMSE-SLE, the SLE is designed to have order $L^{\prime}=12$ and the number of time-varying basis functions $Q^{\prime}=12$. For the case of the MMSE-SDFE, the time-varying FIR feedforward filter is designed to have order $L^{\prime}=12$ and the number of time-varying basis functions $Q^{\prime}=12$, while the time-varying FIR feedback filter is designed to have order $L^{\prime \prime}=L$ and $Q^{\prime \prime}=Q$. The SLE coefficients as well as the SDFE coefficients are computed as explained in [1] for the MMSE-SLE, and in [2] for the MMSE-SDFE. The BEM resolution of the timevarying FIR filters matches that of the channel. QPSK signaling is assumed. We define the SNR as SNR $=(L+1) E_{s} / \sigma_{n}^{2}$, where $E_{s}$ is the QPSK symbol power. As shown in Figure 5, for the case of MMSE-SLE, the BER curve experiences an error floor when $D=165$ for the different scenarios. For the case of $D=95$, we experience an SNR loss of $11.5 \mathrm{~dB}$ for the 


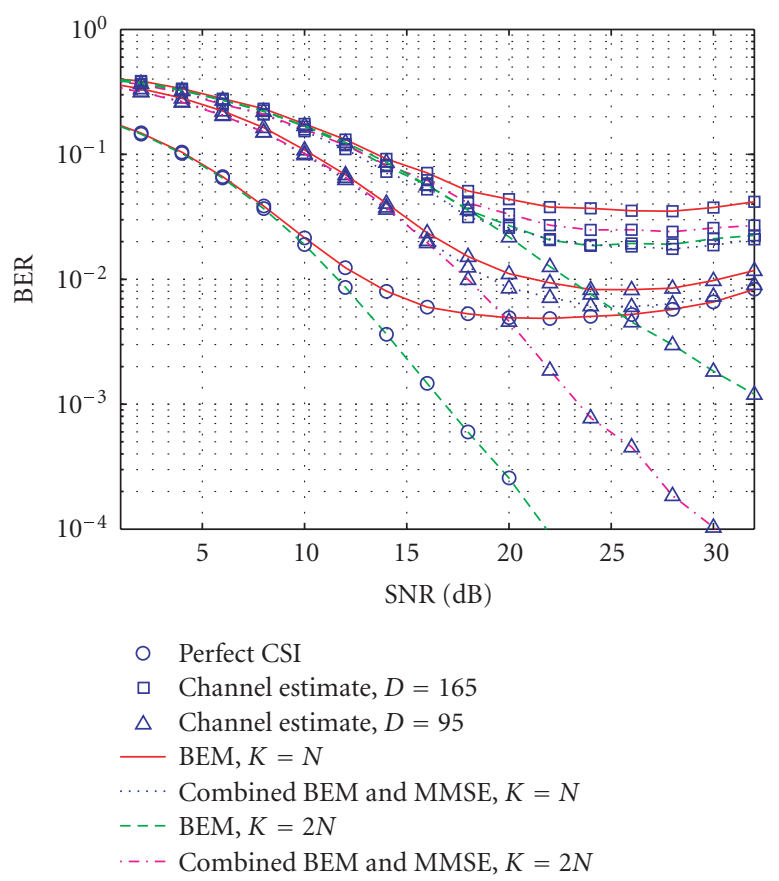

FIGURE 5: BER versus SNR using the MMSE-SLE.

case of $K=2 N$ compared to the case when perfect channel state information (CSI) is known at $B E R=10^{-2}$, while the SNR loss is reduced to $6 \mathrm{~dB}$ for the case of combined BEM and MMSE when $K=2 N$. For $K=N$, both cases (BEM and combined BEM and MMSE) suffer from an error floor. Similar observations can be made for the case of MMSE-SDFE as shown in Figure 6.

\section{(ii) PSAM direct equalization}

We use here the same channel setup. We assume that the training overhead is $50 \%$, that is, we insert a pilot symbol every second symbol. The simulation results are shown in Figure 7 for a BEM resolution $K=N$ as well as for a BEM resolution $K=2 N$. We consider the LS criterion (32) and the regularized LS criterion (35). We choose the equalizer to have a fixed order $L^{\prime}=4$ and variant $Q^{\prime}=4,8$, and 12 . As one can deduce from this figure, the PSAM direct equalization performance relies heavily on the design parameters of the equalizer. As shown in this figure, the performance of the direct PSAM equalizer does not necessarily improve by choosing larger $Q^{\prime}$ and/or $L^{\prime}$, which suggests that there is an optimal $\left(Q^{\prime}, L^{\prime}\right)$ pair. For $Q^{\prime}=4$, the performance of the direct PSAM equalizer for $K=N$ and $K=2 N$ almost coincide, which means for this equalizer setup, BEM oversampling is almost of no effect. The same can be said when $Q^{\prime}=8$. For $Q^{\prime}=12$, the performance of the direct PSAM equalizer for $K=N$ outperforms the one for $K=2 N$. For $K=2 N$ the performance is even worse than for $Q^{\prime}=8$. When $Q^{\prime}=12$, BEM oversampling has a negative impact on the performance. This can be explained by considering the fact that increasing $Q^{\prime}$ on one hand increases the equal-

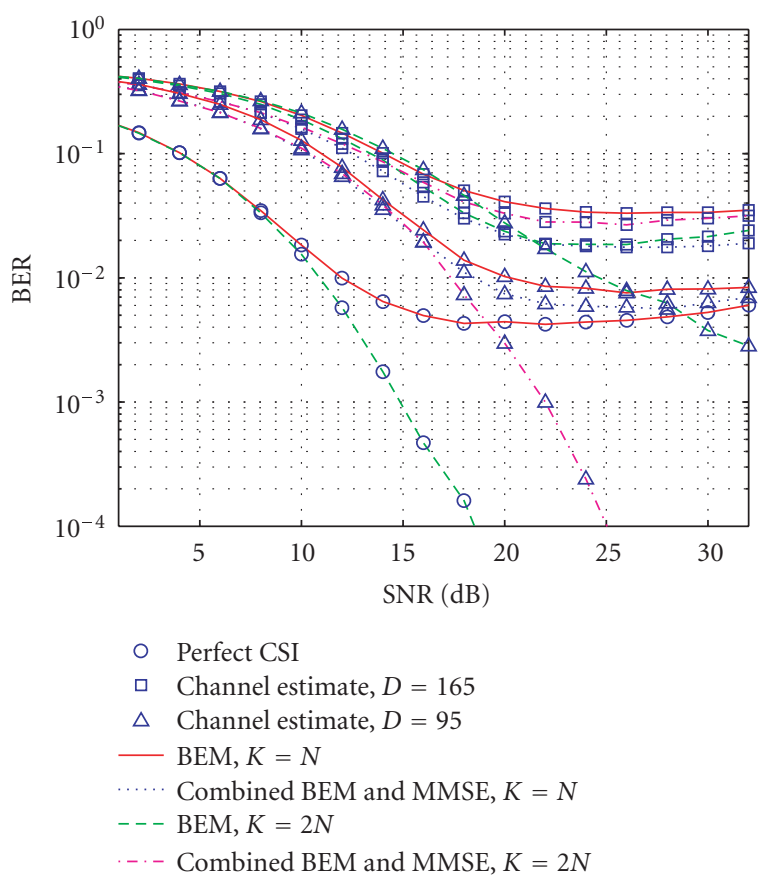

FIgURE 6: BER versus SNR using the MMSE-SDFE.

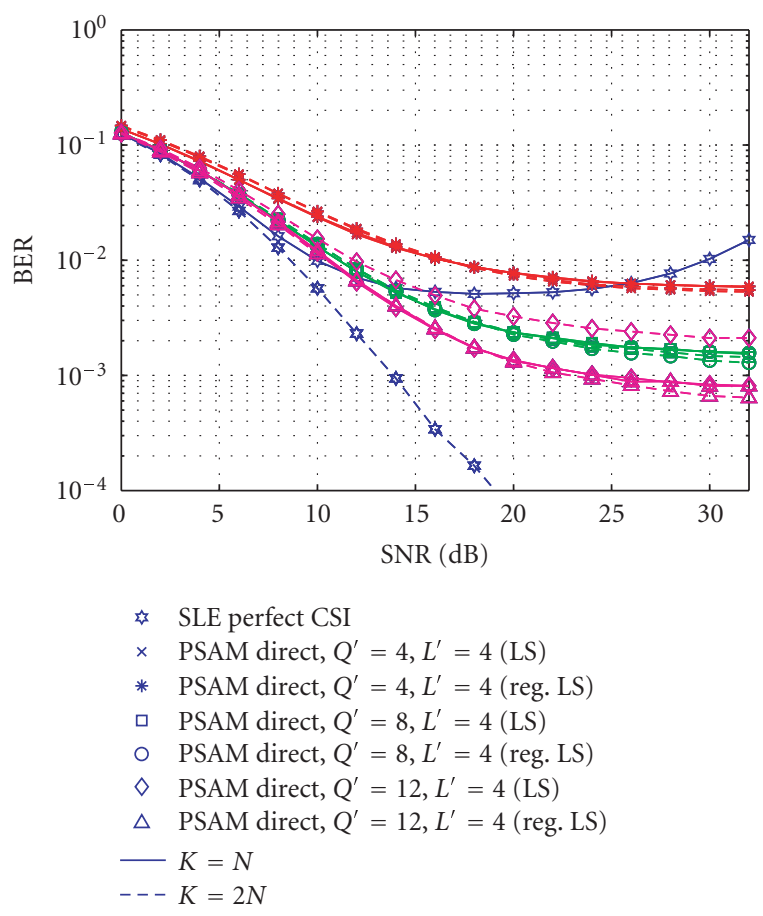

FIGURE 7: BER versus SNR for PSAM-based direct equalization.

izer time-variations which is supposed to have a positive impact on the performance, but on the other hand, it means more parameters have to be identified. For perfect knowledge of CSI, the performance of the time-varying FIR equalizers naturally improves by increasing the number of BEM basis 


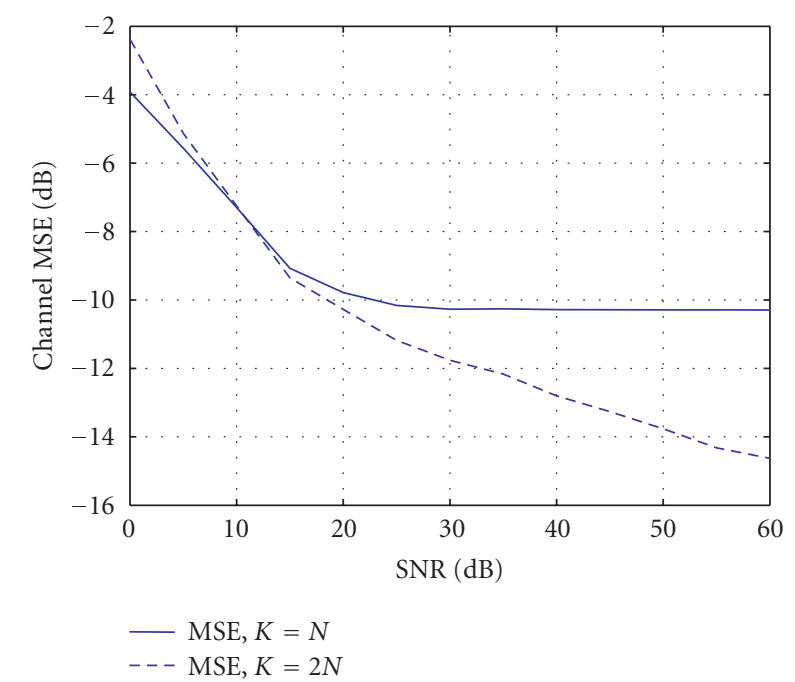

FIGURE 8: MSE versus SNR for the semiblind channel estimation technique.

functions. Note that in the above analysis we did not distinguish between the LS and the regularized LS as they perform almost the same for this setup.

\subsection{Semiblind techniques}

\section{(i) Semiblind channel estimation}

For semiblind channel estimation, we again study MSE versus SNR, where the MSE channel estimation is obtained as in (68). For this case we use the same channel setup as before. We again consider the BEM resolution $K=N$ as well as $K=2 N$. The training part consists of two training clusters of $L+1$ training symbols each. The first one is placed at the beginning of the transmitted block and the other one is placed in the middle of the transmitted block.

First, we study the channel MSE versus SNR for a fixed $\alpha=0.1$. The simulation results are shown in Figure 8 . The MSE channel estimate suffers from an error floor for $K=N$, whereas it shows a slight improvement for $K=2 \mathrm{~N}$ specially for $\mathrm{SNR} \geq 15 \mathrm{~dB}$.

Second, the MSE channel estimate is plotted versus $\alpha$ for a fixed value of SNR $=30 \mathrm{~dB}$. The simulation results are shown in Figure 9. For this channel setup, and for BEM resolutions $K=N$ and $K=2 N$, it is found that the MMSE channel estimate is obtained for $\alpha=0.1$. This actually justifies the choice of $\alpha$ in the first part of the simulations.

\section{(ii) Semiblind direct equalization}

For semiblind direct equalizer estimation, we consider a SIMO system with $N_{r}=4$ receive antennas. We assume a doubly selective channel with Doppler spread of $f_{\max }=100$ and order $L=3$. We use QPSK signaling. We assume the data sequence and the additive noises are mutually uncorrelated and white.

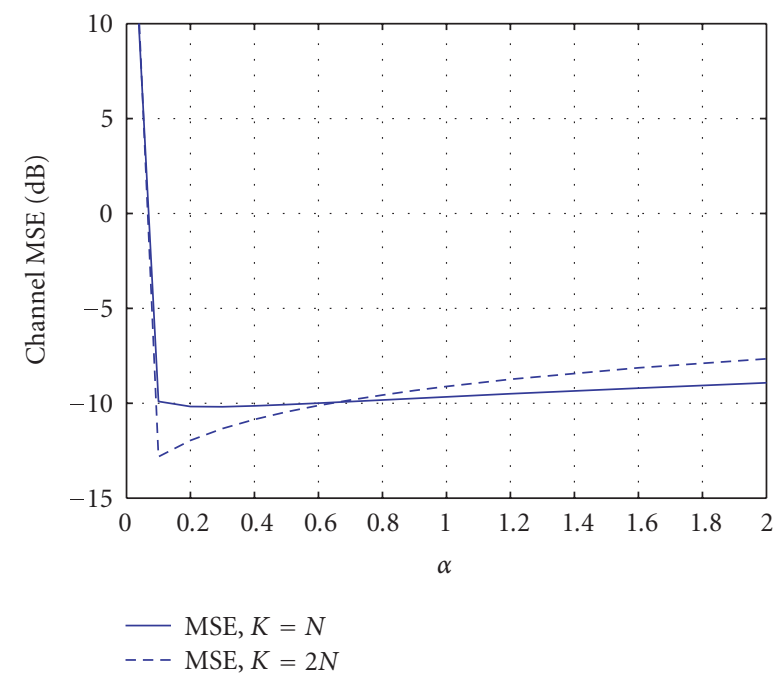

FIGURE 9: MSE versus $\alpha$ for the semiblind channel estimation technique at $\mathrm{SNR}=30 \mathrm{~dB}$.

We consider a time-window of $N T=200 T$. When $N T \leq$ $1 /\left(2 f_{\max }\right)$, which is the case here, an accurate channel model can be obtained by taking $Q=2$. We insert a pilot symbol after every four data symbols (i.e., training overhead of $20 \%$ ). We consider three SLE designs: ideal design where perfect channel state information is assumed to be known at the receiver (see $[1,3])$, the direct training-based design where the equalizer coefficients are obtained directly based on PSAM using the LS (32) as well as the regularized LS (35) criterion (see also [25]), and the direct semiblind design proposed in this paper. For all designs, we assume $Q^{\prime}=2, L^{\prime}=3$, and $d=\left(L+L^{\prime}\right) / 2=3$. For the direct semiblind design we take $K_{1}=L$ and $K_{2}=Q / 2$, that is, we consider the time-shifts $k \in\{0, \ldots, L\}$, and frequency-shifts $p \in\{-Q / 2, \ldots, Q / 2\}$. For the ideal design, we first fit a BEM to the true doubly selective channel over the time window of NT $=200 \mathrm{~T}$, and use the obtained BEM coefficients to design the BEM coefficients of the SLE. The simulation results are shown in Figure 10. From this figure, we can draw the following conclusions:

(1) the direct semiblind design clearly outperforms the direct PSAM when the LS criterion is invoked, where an SNR gain of $16 \mathrm{~dB}$ is observed at $\mathrm{BER}=10^{-2}$,

(2) compared to the regularized direct PSAM, the semiblind has superior performance for the indicated range of SNR for the case of BEM resolution $K=N$. For this case, an SNR gain of $6 \mathrm{~dB}$ is observed at BER $=10^{-2}$. For the case of BEM resolution $K=2 N$, the semiblind technique outperforms the regularized LS direct PSAM for low to moderate values of SNR. The regularized LS direct PSAM slightly outperforms the direct semiblind for SNR > $20 \mathrm{~dB}$. At BER $=10^{-2}$ an SNR gain of $2.5 \mathrm{~dB}$ for the direct semiblind over the regularized LS direct PSAM is observed,

(3) compared to the performance of the MMSE SLE for the perfect CSI, an SNR loss of $5 \mathrm{~dB}$ is observed at $\mathrm{BER}=10^{-2}$ for the direct semiblind design. 


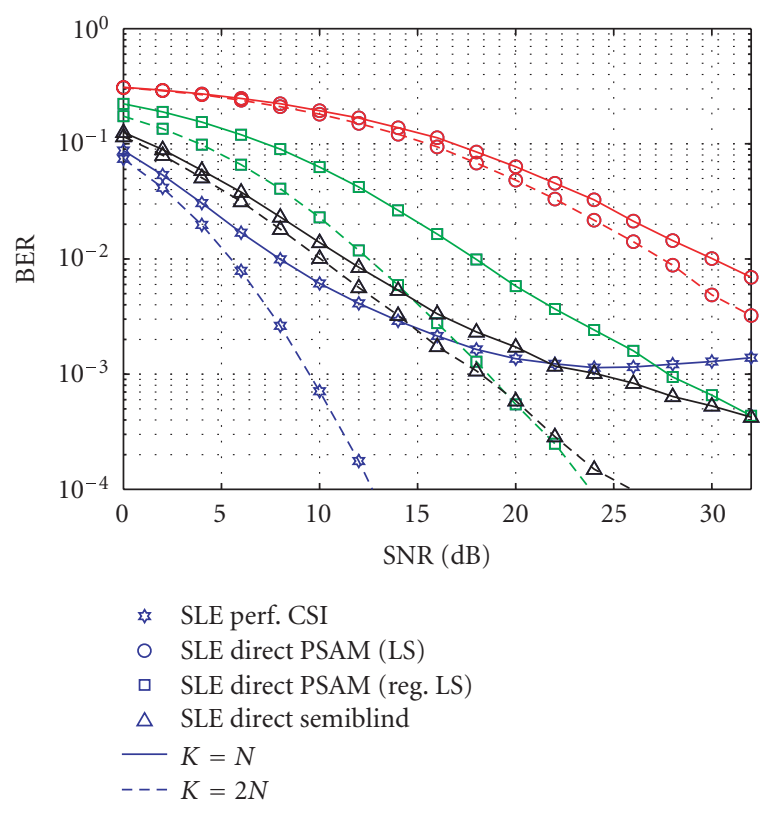

FIGURE 10: Comparison of different SLE designs for doubly selective channels.

\section{CONCLUSIONS}

In this paper, we have proposed channel estimation and direct equalization techniques for transmission over doubly selective channels. In particular, we have proposed PSAM, blind, and semiblind techniques. In PSAM techniques we rely on pilot symbols for channel estimation or direct equalization. We consider the case when the Doppler spectrum is critically sampled $(K=N)$ as well as when the Doppler spectrum is oversampled ( $K \geq r N$ with integer $r>1)$. While in the first case, the estimation scheme suffers from an early error floor due to the large modeling error, the estimation is sensitive to noise in the oversampled case. It has been shown through computer simulations that combining the MMSE-interpolation-based channel estimate with the oversampled BEM significantly improves the channel estimation. We have also shown that the channel estimation step can be skipped by performing direct equalization based on PSAM. For a fixed training scheme, the PSAM-based direct equalizer depends heavily on the equalizer parameters and on the oversampling factor. Whereas, in some cases the oversampled BEM outperforms the critically sampled one, in others the critically sampled outperforms the oversampled BEM. In blind techniques, no training overhead is used to estimate or directly equalize the doubly selective channel. However, they are practically prohibited due to the complexity involved. Semiblind techniques, on the other hand, are obtained by combining the training-based techniques with the blind techniques. Doing so, the scalar ambiguity of the blind techniques is resolved, and the complexity may be greatly reduced especially for the case of direct equalization.

\section{ACKNOWLEDGMENTS}

This research work was carried out at the ESAT Laboratory of the Katholieke Universiteit Leuven, in the frame of the Belgian State, Prime Minister's Office-Federal Office for Scientific, Technical, and Cultural Affairs-Interuniversity Poles of Attraction Programme (2002-2007), P5/11 "mobile multimedia communication systems and networks", the Concerted Research Action GOA-MEFISTO-666 (Mathematical Engineering for Information and Communication Systems Technology) of the Flemish Government, and Research Project FWO no. G.0196.02 "design of efficient communication techniques for wireless time-dispersive multiuser MIMO systems". The scientific responsibility is assumed by its authors. The first author was partly supported by the Palestinian European Academic Cooperation in Education (PEACE) Programme. The second author was supported in part by the NWO-STW under the VIDI Program (DTC.6577).

\section{REFERENCES}

[1] I. Barhumi, G. Leus, and M. Moonen, "Time-varying FIR equalization for doubly selective channels," IEEE Transactions on Wireless Communications, vol. 4, no. 1, pp. 202-214, 2005.

[2] I. Barhumi, G. Leus, and M. Moonen, "Time-varying FIR decision feedback equalization of doubly-selective channels," in Proceedings of IEEE Global Telecommunications Conference (GLOBECOM '03), vol. 4, pp. 2263-2268, San Francisco, Calif, USA, December 2003.

[3] G. Leus, I. Barhumi, and M. Moonen, "Low-complexity serial equalization of doubly selective channels," in Proceedings of 6th Baiona Workshop on Signal Processing in Communications, pp. 69-74, Baiona, Spain, September 2003.

[4] I. Barhumi, G. Leus, and M. Moonen, "Per-tone equalization for OFDM over doubly-selective channels," in Proceedings of the IEEE International Conference on Communications, vol. 5, pp. 2642-2647, Paris, France, June 2004.

[5] T. Thomas and F. Vook, "Multi-user frequency-domain channel identification, interference suppression, and equalization for time-varying broadband wireless communications," in Proceedings of the 1st IEEE Sensor Array and Multichannel Signal Processing Workshop (SAM '00), pp. 444-448, Cambridge, Mass, USA, March 2000.

[6] X. Ma, G. B. Giannakis, and S. Ohno, "Optimal training for block transmissions over doubly selective wireless fading channels," IEEE Transactions on Signal Processing, vol. 51, no. 5, pp. 1351-1366, 2003.

[7] G. Xu, H. Liu, L. Tong, and T. Kailath, "Least-squares approach to blind channel identification," IEEE Transactions on Signal Processing, vol. 43, no. 12, pp. 2982-2993, 1995.

[8] D. Gesbert, P. Duhamel, and S. Mayrargue, "On-line blind multichannel equalization based on mutually referenced filters," IEEE Transactions on Signal Processing, vol. 45, no. 9, pp. 2307-2317, 1997.

[9] G. B. Giannakis and C. Tepedelenlioğlu, "Direct blind equalizers of multiple FIR channels: a deterministic approach," IEEE Transactions on Signal Processing, vol. 47, no. 1, pp. 62-74, 1999.

[10] E. Moulines, P. Duhamel, J.-f. Cardoso, and S. Mayrargue, "Subspace methods for the blind identification of 
multichannel FIR filters," IEEE Transactions on Signal Processing, vol. 43, no. 2, pp. 516-525, 1995.

[11] K. Abed-Meraim, P. Loubaton, and E. Moulines, "A subspace algorithm for certain blind identification problems," IEEE Transactions on Information Theory, vol. 43, no. 2, pp. 499511, 1997.

[12] K. Abed-Meraim, W. Qiu, and Y. Hua, "Blind system identification," Proceedings of the IEEE, vol. 85, no. 8, pp. 1310-1322, 1997.

[13] X. Wang and H. V. Poor, "Blind multiuser detection: a subspace approach," IEEE Transactions on Information Theory, vol. 44, no. 2, pp. 677-690, 1998.

[14] L. Tong and S. Perreau, "Multichannel blind identification: from subspace to maximum likelihood methods," Proceedings of the IEEE, vol. 86, no. 10, pp. 1951-1968, 1998.

[15] G. B. Giannakis, Y. Hua, P. Stoica, and L. Tong, Eds., Signal Processing Advances in Wireless \& Mobile Communications: Trends in Single and Multi-User Systems, Prentice-Hall, New York, NY, USA, 2001.

[16] G. B. Giannakis and C. Tepedelenlioğlu, "Basis expansion models and diversity techniques for blind identification and equalization of time-varying channels," Proceedings of the IEEE, vol. 86, no. 10, pp. 1969-1986, 1998.

[17] H. Liu and G. B. Giannakis, "Deterministic approaches for blind equalization of time-varying channels with antenna arrays," IEEE Transactions on Signal Processing, vol. 46, no. 11, pp. 3003-3013, 1998.

[18] J. K. Tugnait, "Linear prediction error method for blind identification of time-varying channels: theoretical results," in Proceedings of IEEE International Conference on Acoustics, Speech and Signal Processing (ICASSP '01), vol. 4, pp. 2125-2128, Salt Lake City, Utah, USA, May 2001.

[19] J. K. Tugnait and W. Luo, "Blind identification of time-varying channels using multistep linear predictors," IEEE Transactions on Signal Processing, vol. 52, no. 6, pp. 1739-1749, 2004.

[20] W. C. Jakes, Ed., Microwave Mobile Communications, John Wiley \& Sons, New York, NY, USA, 1974.

[21] A. M. Sayeed and B. Aazhang, "Joint multipath-doppler diversity in mobile wireless communications," IEEE Transactions on Communications, vol. 47, no. 1, pp. 123-132, 1999.

[22] X. Ma and G. B. Giannakis, "Maximum-diversity transmissions over doubly selective wireless channels," IEEE Transactions on Information Theory, vol. 49, no. 7, pp. 1832-1840, 2003.

[23] G. Leus, S. Zhou, and G. B. Giannakis, "Orthogonal multiple access over time- and frequency-selective channels," IEEE Transactions on Information Theory, vol. 49, no. 8, pp. 19421950, 2003.

[24] A. P. Kannu and P. Schniter, "Capacity analysis of MMSE pilotaided transmission for doubly selective channels," in Proceedings of the IEEE 6th Workshop on Signal Processing Advances in Wireless Communications (SPAWC '05), vol. 2005, pp. 801805, New York, NY, USA, June 2005.

[25] J. K. Cavers, "An analysis of pilot symbol assisted modulation for Rayleigh fading channels [mobile radio]," IEEE Transactions on Vehicular Technology, vol. 40, no. 4, pp. 686-693, 1991.

[26] I. Barhumi, G. Leus, and M. Moonen, "Frequency-domain equalization for OFDM over doubly selective channels," in Proceedings of 6th Baiona Workshop on Signal Processing in Communications, pp. 103-107, Baiona, Spain, September 2003.
[27] G. Leus, "On the estimation of rapidly time-varying channels," in Proceedings of the European Signal Processing Conference (EUSIPCO '04), pp. 2227-2230, Vienna, Austria, September 2004.

[28] D. W. Clarke, "Generalized-least-squares estimation of the parameters of a dynamic model," in Proceedings of 1st IFAC Symposium on Identification and System Parameter Estimation, Prague, Czechoslovakia, 1967.

[29] G. Leus and M. Moonen, "Deterministic subspace based blind channel estimation for doubly selective channels," in Proceedings of the 4th IEEE Workshop on Signal Processing Advances in Wireless Communications (SPAWC '03), pp. 210-214, Rome, Italy, June 2003.

[30] S. Ratnavel, A. Paulraj, and A. G. Constantinides, "MMSE space-time equalization for GSM cellular systems," in Proceedings of IEEE 46th Vehicular Technology Conference (VTC '96), vol. 1, pp. 331-335, Atlanta, Ga, USA, April-May 1996.

Imad Barhumi was born in Palestine in 1972. He received the B.S. degree in electrical engineering from Birzeit University, Birzeit, Palestine, in 1996, the M.S. in telecommunications from University of Jordan, Amman, Jordan, in 1999, and the Ph.D. degree from the Katholieke Universiteit Leuven, Leuven, Belgium, in 2005. From 1999 to 2000, he was with the Electrical Engineering Department at Birzeit Uni-

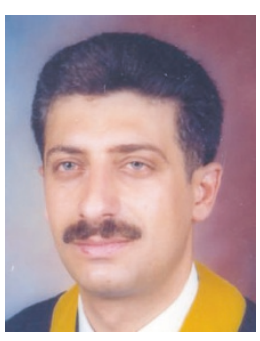
versity as a Lecturer. After his Ph.D. graduation, he served one year as a Postdoctoral Research Fellow at the Electrical Engineering Department of the Katholieke Universiteit Leuven. Currently, he is an Assistant Professor with the Electrical Engineering Department of the United Arab Emirates University, Al-Ain, UAE. His research interests are in the area of signal processing for mobile and wireless telecommunications.

Geert Leus was born in Leuven, Belgium, in 1973. He received the Electrical Engineering degree and the Ph.D. degree in applied sciences from the Katholieke Universiteit Leuven, Belgium, in June 1996 and May 2000, respectively. He has been a Research Assistant and a Postdoctoral Fellow of the Fund for Scientific Research-Flanders, Belgium, from October 1996 till September 2003. During that period, he was affiliated with

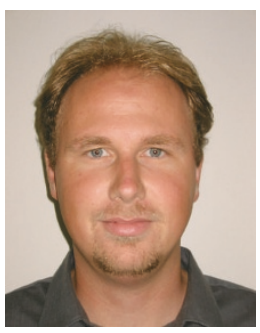
the Electrical Engineering Department of the Katholieke Universiteit Leuven, Belgium. Currently, he is an Assistant Professor at the Faculty of Electrical Engineering, Mathematics and Computer Science of the Delft University of Technology, The Netherlands. During the summer of 1998, he visited Stanford University, and from March 2001 till May 2002, he was a Visiting Researcher and Lecturer at the University of Minnesota. His research interests are in the area of signal processing for communications. He received a 2002 IEEE Signal Processing Society Young Author Best Paper Award and a 2005 IEEE Signal Processing Society Best Paper Award. $\mathrm{He}$ is a Member of the IEEE Signal Processing for Communications Technical Committee, and an Associate Editor for the IEEE Transactions on Signal Processing and the EURASIP Journal on Applied Signal Processing. In the past, he has served on the editorial board of the IEEE Signal Processing Letters and the IEEE Transactions on Wireless Communications. 
Marc Moonen received the Electrical Engineering degree and the Ph.D. degree in applied sciences from the Katholieke Universiteit Leuven, Leuven, Belgium, in 1986 and 1990, respectively. Since 2000, he has been an Associate Professor at the Electrical Engineering Department of Katholieke Universiteit Leuven, where he is currently heading a research team of 16 Ph.D. candidates and postdocs, working in the area

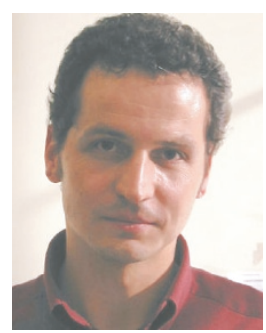
of signal processing for digital communications, wireless ommunications, DSL, and audio signal processing. He received the 1994 K.U.Leuven Research Council Award, the 1997 Alcatel Bell (Belgium) Award (with Piet Vandaele), and was a 1997 "Laureate of the Belgium Royal Academy of Science." He was Chairman of the IEEE Benelux Signal Processing Chapter (1998-2002), and is currently a EURASIP AdCom Member (European Association for Signal Processing, 2000 to present). He is Editor-in-Chief for the "EURASIP Journal on Applied Signal Processing" (2003 to present), and a Member of the editorial board of "Integration, the VLSI Journal," "IEEE Transactions on Circuits and Systems II" (2002-2003), "EURASIP Journal on Wireless Communications and Networking," and "IEEE Signal Processing Magazine." 